\title{
UNIQUENESS AND EXISTENCE OF VISCOSITY SOLUTIONS OF GENERALIZED MEAN CURVATURE FLOW EQUATIONS
}

\author{
YUN-GANG CHEN, YOSHIKAZU GIGA \& SHUN'ICHI GOTO
}

\section{Introduction}

This paper treats degenerate parabolic equations of second order

$$
u_{t}+F\left(\nabla u, \nabla^{2} u\right)=0
$$

related to differential geometry, where $\nabla$ stands for spatial derivatives of $u=u(t, x)$ in $x \in \mathbb{R}^{n}$, and $u_{t}$ represents the partial derivative of $u$ in time $t$. We are especially interested in the case when (1.1) is regarded as an evolution equation for level surfaces of $u$. It turns out that (1.1) has such a property if $F$ has a scaling invariance

$$
F(\lambda p, \lambda X+\sigma p \otimes p)=\lambda F(p, X), \quad \lambda>0, \sigma \in \mathbb{R},
$$

for a nonzero $p \in \mathbb{R}^{n}$ and a real symmetric matrix $X$, where $\otimes$ denotes a tensor product of vectors in $\mathbb{R}^{n}$. We say (1.1) is geometric if $F$ satisfies (1.2). A typical example is

$$
u_{t}-|\nabla u| \operatorname{div}(\nabla u /|\nabla u|)=0,
$$

where $\nabla u$ is the (spatial) gradiant of $u$. Here $\nabla u /|\nabla u|$ is a unit normal to a level surface of $u$, so $\operatorname{div}(\nabla u /|\nabla u|)$ is its mean curvature unless $\nabla u$ vanishes on the surface. Since $u_{t} /|\nabla u|$ is a normal velocity of the level surface, (1.3) implies that a level surface of solution $u$ of (1.3) moves by its mean curvature unless $\nabla u$ vanishes on the surface. We thus call (1.3) the mean curvature flow equation in this paper.

The motion of a closed (hyper)surface in $\mathbb{R}^{n}$ by its mean curvature has been studied by many authors [1], [3], [4], [8], [10], [12], [14], [15]. Such a motion is also important in the singular perturbation theory related to

Received August 1, 1989 and, in revised form, March 5, 1990. The first author is on leave from and was partially supported by Nankai Institute of Mathematics, Tianjin, China. The second author was partially supported by the Japan Ministry of Education, Science and Culture through grants No. 01740076 and 01540092 for scientific research. 
phase transition phenomena (see [13], [23] and references therein). However, so far whole unique evolution families of surfaces were only constructed under geometric restrictions on initial surfaces such as convexity [10], [14], except $n=2$ [3], [12]. When $n=2$, M. Grayson [12] has shown that any embedded curve moved by its curvature never becomes singular unless it shrinks to a point. However, when $n \geq 3$ even embedded surfaces may develop singularities before it shrinks to a point. A barbell with a long, thin handle actually becomes singular in the middle in short time.

Our goal is to construct whole unique evolution families of surfaces even after the time when there appear singularities. Contrary to other authors (except [4]) we avoid parametrization and rather understand surfaces as level sets of solutions $u$ of (1.3). We first study the initial value problem for a geometric, degenerate parabolic equation (1.1) with

$$
u(0, x)=a(x) \in C_{\alpha}\left(\mathbb{R}^{n}\right)
$$

for some constant $\alpha$, where $C_{\alpha}(A)$ is the set of continuous functions $a$ in $A$ such that $a-\alpha$ is compactly supported in $A$. Recently P. L. Lions [22] introduced a class of weak solutions for degenerate elliptic equations of second order so that a comparison principle holds. Such solutions are called viscosity solutions, and a general theory is established by $R$. Jensen [19] and H. Ishii [17] (see also [6] for simplification). For a large class of geometric, degenerate parabolic equations including (1.3) we construct a unique global viscosity solution $u_{a}$ in $C_{\alpha}\left([0, T] \times \mathbb{R}^{n}\right)$ of (1.1) and (1.4) for every $T>0$. Since our $F(p, X)$ is singular at $p=0$, as is observed in (1.3), even uniqueness of viscosity solutions does not follow directly from results in [17], [19]. We are forced to extend their theory to our situation. Existence of viscosity solutions is based on Perron's method discussed in [16], [17]. We construct viscosity sub- and supersolutions of (1.1) and (1.4) and obtain the viscosity solution $u_{a}$.

We now turn to the study of level surfaces of a viscosity solution $u_{a}$ of (1.1) and (1.4). Let $\Gamma(t)$ be the $\gamma$-level surface of $u_{a}(t, \cdot)$ and let $D(t)$ be the set of $x \in \mathbb{R}^{n}$ such that $u_{a}(t, x)>\gamma$, where $\gamma>\alpha$. We call $u_{a}(t, \cdot)$ a defining function of $(\Gamma(t), D(t))$. When (1.1) is geometric and degenerate parabolic, using (1.2) we show that if $u$ is a viscosity sub(super)solution of $(1.1)$, so is $\theta(u)$ provided that $\theta$ is continuous and nondecreasing. This is proved in $\S 5$ and the proof depends on Jensen's lemma on semiconvex functions [19]. By this property of $(1.1)$ we prove that the family $(\Gamma(t), D(t))(t \geq 0)$ is uniquely determined by $(\Gamma(0), D(0))$ and is independent of a choice of the defining functions $a$ of $(\Gamma(0), D(0))$. 
We call this evolution family $(\Gamma(t), D(t))(t \geq 0)$ a solution family of (1.1) with initial data $(\Gamma(0), D(0))$. If $D(0)$ is a bounded open set and $\Gamma(0)\left(\subset \mathbb{R}^{n} \backslash D(0)\right)$ is a compact set containing the boundary $\partial D(0)$, then, evidently, there is a defining function $a \in C_{\alpha}\left(\mathbb{R}^{n}\right)$ of $(\Gamma(0), D(0))$. Existence of a viscosity solution $u_{a}$ of (1.1) and (1.4) now yields a unique global solution family $(\Gamma(t), D(t))(t \geq 0)$ of (1.1) for a given initial data $(\Gamma(0), D(0))$. In particular for the mean curvature flow equation (1.3) we construct a whole unique evolution family $\Gamma(t)$ moved by its mean curvature. Since $\Gamma(t)$ may not be regular, here its mean curvature is interpreted in some weak sense. By our comparison it is also proved that $(\Gamma(t), D(t))$ becomes empty in finite time provided that it is a solution family of (1.3) when $n \geq 2$. This extends a result of $\mathrm{G}$. Huisken [14] because no geometric assumption of $\Gamma(0)$ is required in our approach. In [14] Huisken proved that $\Gamma(t)$ becomes extinct in finite time provided that $\Gamma(0)$ is a uniformly convex $C^{2}$ surface in $\mathbb{R}^{n}(n \geq 3)$; for $n=2$, see [10]. We also note that we need no regularity of $\Gamma(0)$. In [4] K. A. Brakke tried to construct a global evolution family $\Gamma(t)$ moved by its mean curvature by using geometric measure theory. However, his varifold solution is too weak to be regarded as an evolution of subsets in $\mathbb{R}^{n}$, and his solution may not be unique.

Our analysis works for a large class of geometric, degenerate parabolic equations (1.1) other than the mean curvature flow equation (1.3). Important examples generalizing (1.3) are

$$
u_{t}-|\nabla u| \operatorname{div}(\nabla u /|\nabla u|)-\nu|\nabla u|=0, \quad \nu \in \mathbb{R},
$$

and its anisotropic version (cf. [13])

$$
u_{t}-|\nabla u| \sum_{i=1}^{n} \frac{\partial}{\partial x_{i}}\left(\frac{\partial H}{\partial p_{i}}\left(\frac{\nabla u}{|\nabla u|}\right)\right)-\beta\left(\frac{\nabla u}{|\nabla u|}\right)|\nabla u|=0,
$$

where $H \in C^{2}\left(\mathbb{R}^{n} \backslash\{0\}\right)$ is convex, positively homogeneous of degree one and $\beta$ is continuous on a unit sphere in $\mathbb{R}^{n}$. In (1.5) one observes that the motion of $\Gamma(t)$ by constant speed $\nu$ is also considered as well as by the mean curvature. When $n=2$, a global evolution family of curves $\Gamma(t)$ (even not embedded) moved by (1.6) with $\beta=0$ is essentially constructed in [3] by a completely different method-parametrization of $\Gamma(t)$. In [3] strict convexity of $H$ is also assumed. As is described in later sections our analysis works even when $F$ depends on $t$. However, the case where $F$ depends on $x$ is not studied in the present paper.

This paper is organized as follows. $\S 2$ begins with the definition of viscosity sub- and supersolutions and treats its basic properties. Most of the 
results in this section are more or less known to specialists. However, the proof in our context is not explicitly written in the literature, and ideas of the proof are scattered in various literature, so we include the proof both for completeness and for the reader's convenience. In $\S 3$ we state a parabolic version of Ishii's lemma [17], [18], which is a key to establishing the comparison principle for viscosity solutions. Our results extend Proposition IV.1 in [18]. $\S 4$ establishes comparison results on viscosity sub- and supersolutions of degenerate parabolic equations, including (1.1) in a bounded domain even when $F=F(p, X)$ may not be continuous at $p=0$. This extends the comparison results in [17], [18], where $F$ is assumed to be continuous. $\S 5$ begins with the definition of geometric equations, and there we show that geometric, degenerate elliptic equations are invariant under an (orientation-preserving) change of a dependent variable in the viscosity sense, as is mentioned in the fourth paragraph of the Introduction. We use approximation and Jensen's lemma in [19] on semiconvex functions to show this fact. In $\S \S 6$ and 7 we consider the initial-value problem of the geometric, degenerate parabolic equation (1.1) with (1.4). §6 establishes the unique global existence of viscosity solutions for a large class of (1.1) by Perron's method. Using results in $\S 6$, we construct a solution family $(\Gamma(t), D(t))$ of $(1.1)$ for an arbitrary initial data $(\Gamma(0), D(0))$. The main body of this paper consists of $\S \S 4-7$ preceded by preliminary $\S \S 2$ and 3. This paper is written so that no previous knowledge of viscosity solutions in [16], [17], [18], [19], [22], [24] is required.

The results in this paper have been announced in [5].

After this work was completed, we were informed of a recent work of L. C. Evans and J. Spruck [9] closely related to ours. They also proved the existence of a unique viscosity solution and studied various properties of the level surfaces $\Gamma(t)$ of the solution, but only for the mean curvature flow equation (1.3). They showed that $\Gamma(t)$ is determined only by $\Gamma(0)$, which is not expected for general geometric equations having first-order terms such as (1.5) with $\nu \neq 0$. We also learned of works of S. Osher and J. A. Sethian [25] and Sethian [26] giving numerical algorithms for evolutions of surfaces with curvature-dependent speed. Their viewpoint of an evolution of surfaces is the same as ours. They regarded them as level surfaces of solutions of parabolic equations of second order.

We are grateful to Professor Hitoshi Ishii and Professor Robert Kohn for informing us of several recent works related to ours. We are also grateful to Professor L. Craig Evans for sending us his latest manuscript with J. Spruck [9] before its publication. 


\section{Basic properties of viscosity solutions}

We recall the definition of a viscosity solution and collect some of its properties here. This section is almost paralleled to $\S 2$ in [17], although the situation is slightly different.

For a sequence of functions $h_{k}: L \rightarrow \mathbb{R}\left(L \subset \mathbb{R}^{d}\right)(k=1,2, \ldots)$ we associate its $\Gamma^{-}$-limit

defined by

$$
\lim _{k \rightarrow \infty} h_{k}: \bar{L} \rightarrow \widetilde{\mathbb{R}}=\mathbb{R} \cup\{ \pm \infty\}
$$

$$
\lim _{k \rightarrow \infty} h_{k}(x)=\lim _{\substack{k \rightarrow \infty \\
\varepsilon \downarrow 0}} \inf _{\substack { l \geq k \\
\begin{subarray}{c}{|x-y|<\varepsilon \\
y \in L{ l \geq k \\
\begin{subarray} { c } { | x - y | < \varepsilon \\
y \in L } }\end{subarray}} h_{l}(y) \text { for } x \in \bar{L},
$$

where $\bar{L}$ denotes the closure of $L$ in $\mathbb{R}^{d}$. When $h_{k}=h$ for all $k$, the $\Gamma^{-}$-limit $\lim _{* k \rightarrow \infty} h_{k}$ is called the lower semicontinuous (l.s.c.) relaxation of $h$ to $\bar{L}$ and is denoted by $h_{*}$. It is easy to see

$$
h_{*}(x)=\lim _{\varepsilon \downarrow 0} \inf _{\substack{|x-y|<\varepsilon \\ y \in L}} h(y), \quad x \in \bar{L} .
$$

The upper semicontinuous (u.s.c.) relaxation of $h$ to $\bar{L}$ is defined by $h^{*}=-(-h)_{*}$. The concept of $\Gamma^{-}$-limit and the relaxations was introduced by E. De Giorgi [11] and it is important, for example in the calculus of variation.

For $A \subset \mathbb{R}^{N}$ we consider a dense subset $W$ of $J(A)=A \times \mathbb{R} \times \mathbb{R}^{N} \times$ $S^{N \times N}$, where $S^{N \times N}$ denotes the space of $N \times N$ real symmetric matrices. Let $E=E(y, s, q, Y)$ be a function from $W$ to $\mathbb{R}$. Since $W$ is dense in $J(A)$, the relaxations $E_{*}$ and $E^{*}$ are defined in $J(A)$ with value in $\widetilde{\mathbb{R}}$.

Definition 2.1. A function $u=u(y): A \rightarrow \mathbb{R}$ is called a viscosity subsolution (supersolution, respectively) of the equation

$$
E\left(y, u, D u, D^{2} u\right)=0 \text { in } A
$$

if $u^{*}<\infty\left(u_{*}>-\infty\right.$, resp. $)$ in $A$, and for each pair $\phi \in C^{2}(A)$ and $\bar{y} \in A$ satisfying $\max _{A}\left(u^{*}-\phi\right)=\left(u^{*}-\phi\right)(\bar{y})\left(\min _{A}\left(u_{*}-\phi\right)=\left(u_{*}-\phi\right)(\bar{y})\right.$, resp.) it holds that

$$
\begin{gathered}
E_{*}\left(\bar{y}, u^{*}(\bar{y}), D \phi(\bar{y}), D^{2} \phi(\bar{y})\right) \leq 0 \\
\left(E^{*}\left(\bar{y}, u_{*}(\bar{y}), D \phi(\bar{y}), D^{2} \phi(\bar{y})\right) \geq 0, \text { resp. }\right) .
\end{gathered}
$$


Here $D u=\left(\partial u / \partial y_{1}, \cdots, \partial u / \partial y_{N}\right)$, and $D^{2} u$ denotes the Hessian matrix of $u$. By $\phi \in C^{2}(A)$ we mean that $\phi$ has a $C^{2}$ extension $\tilde{\phi}$ in an open neighborhood of $A$.

When $u$ has the second differential at $\bar{y} \in A$ it is easy to see that

$$
E_{*}\left(\bar{y}, u(\bar{y}), D u(\bar{y}), D^{2} u(\bar{y})\right) \leq 0
$$

if $u$ is a viscosity subsolution. A similar result holds for a viscosity supersolution. A function $u=u(y)$ is called a viscosity solution of (2.1) if it is both a viscosity sub- and supersolution of (2.1).

Our definition has a meaning for a wider class of $E$ than in [7], [17], since we do not assume $W=J(A)$ here. We often suppress the word "viscosity", except in statements of theorems, since all solutions in this paper are considered in the viscosity sense.

In what follows we always assume that $A$ is locally compact. We give below three basic properties of subsolutions.

Proposition 2.2. Let $S$ be a nonempty family of a subsolution of (2.1) and let $u$ be a function defined on $A$ by

$$
u(y)=\sup \{v(y) ; v \in S\} \quad \text { for } y \in A .
$$

Suppose $u^{*}(y)<\infty$ for $y \in A$. Then $u$ is a subsolution of (2.2).

Proposition 2.3 (Existence). Suppose that $E$ is degenerate elliptic, i.e.,

$$
E(y, s, q, Y) \leq E(y, s, q, Z) \text { in } W \text { if } Y \geq Z \text {. }
$$

Let $f$ and $g: A \rightarrow \mathbb{R}$ be respectively a sub- and supersolution of (2.1). Suppose $f \leq g$ in $A$. Then there exists a solution $u$ of (2.1) satisfying $f \leq u \leq g$ in $A$.

Proposition 2.4 (Stability). Let $E, E_{k}: W \rightarrow \mathbb{R}$ and $u_{k}$ be a subsolution of

$$
E_{k}\left(y, u_{k}, D u_{k}, D^{2} u_{k}\right)=0 \text { in } A \text {, }
$$

$k=1,2, \cdots$. Assume $\lim _{* \rightarrow \infty} E_{k} \geq E_{*}$ and $u_{k}$ converges to a function $u: A \rightarrow \mathbb{R}$ uniformly in each compact subset of $A$. Then, $u$ is a subsolution of (2.1) with this $E$.

When $E$ is continuous in $W=J(A)$, Propositions 2.2 and 2.3 are studied in [17]. Existence of the solution (Proposition 2.3) is proved by Perron's method as in [16]. Although the proof is actually written for first order equations in [16], it still works in our situation with minor modifications. However, we give proofs both of Propositions 2.2 and 2.3 for the reader's convenience and for completeness because the proof for second order equations is not explicitly written in the literature. 
We also give the proof of Proposition 2.4. The stability is often proved under stringent assumptions that $E_{k}, E: J(A) \rightarrow \mathbb{R}$ are continuous and that $E_{k} \rightarrow E$ uniformly on every compact subset of $J(A)$ (cf. [22]).

Proof of Proposition 2.2. First, we choose a function $\phi \in C^{2}(A)$ and a point $\bar{y} \in A$ such that

$$
\max _{A}\left(u^{*}-\phi\right)=\left(u^{*}-\phi\right)(\bar{y}),
$$

and then fix $\phi$ and $\bar{y}$. Here we can assume $\left(u^{*}-\phi\right)(\bar{y})=0$ without loss of generality, since the function $\phi(y)$ can be replaced by $\phi(y)+\left(u^{*}-\phi\right)(\bar{y})$. Putting $\psi(y)=\phi(y)+|y-\bar{y}|^{4}$, we see that $u^{*}-\psi$ attains its strict maximum in $A$ at $\bar{y}$. Then

$$
\left(u^{*}-\psi\right)(y)+|y-\bar{y}|^{4}=\left(u^{*}-\phi\right)(y) \leq\left(u^{*}-\phi\right)(\bar{y})=\left(u^{*}-\psi\right)(\bar{y})=0
$$

yields

$$
\left(u^{*}-\psi\right)(y) \leq-|y-\bar{y}|^{4} \text { for } y \in A .
$$

By the definition of $u^{*}$, there is a sequence $\left\{x_{k}\right\} \subset A$ such that $x_{k} \rightarrow$ $\bar{y}(k \rightarrow \infty)$ and

$$
\lim _{k \rightarrow \infty} a_{k}=\left(u^{*}-\phi\right)(\bar{y})=0
$$

with $a_{k}=(u-\phi)\left(x_{k}\right)$. Since $u(y)=\sup \{v(y) ; v \in S\}$, there exists a sequence $\left\{v_{k}\right\} \subset S$ such that $v_{k}\left(x_{k}\right)>u\left(x_{k}\right)-1 / k(k=1,2, \cdots)$. This implies

$$
\left(v_{k}^{*}-\phi\right)\left(x_{k}\right) \geq\left(v_{k}-\phi\right)\left(x_{k}\right)>a_{k}-1 / k .
$$

Since $v_{k} \leq u$, by $(2.2)$ we get

$$
\left(v_{k}^{*}-\psi\right)(y) \leq-|y-\bar{y}|^{4} \text { for } y \in A .
$$

Since $A$ is locally compact, there is a compact neighborhood of $\bar{y}$ which we denote by $B$. Because the function $\left(v_{k}^{*}-\phi\right)(x)$ is u.s.c. and has an upper bound, it attains its maximum in $B$ at a point $y_{k} \in B$. We thus conclude

$$
a_{k}-1 / k<\left(v_{k}^{*}-\psi\right)\left(x_{k}\right) \leq\left(v_{k}^{*}-\psi\right)\left(y_{k}\right) \leq-\left|y_{k}-\bar{y}\right|^{4} .
$$

This implies $y_{k} \rightarrow \bar{y}(k \rightarrow \infty)$. Indeed, if not, we would have

$$
0=\lim _{k \rightarrow \infty}(u-\phi)\left(x_{k}\right) \leq \limsup _{k \rightarrow \infty}\left(1 / k-\left|y_{k}-\bar{y}\right|^{4}\right)<0,
$$

which is a contradiction. 
By (2.3) we can see $\lim _{k \rightarrow \infty}\left(v_{k}^{*}-\psi\right)\left(y_{k}\right)=0$, and hence

$$
\lim _{k \rightarrow \infty} v_{k}^{*}\left(y_{k}\right)=\lim _{k \rightarrow \infty} \psi\left(y_{k}\right)=\psi(\bar{y})=u^{*}(\bar{y}) \text {. }
$$

Since $v_{k}$ is a subsolution we see

$$
E_{*}\left(y_{k}, v_{k}^{*}\left(y_{k}\right), D \psi\left(y_{k}\right), D^{2} \psi\left(y_{k}\right)\right) \leq 0 .
$$

Since $E_{*}$ is 1.s.c. and $D \psi(\bar{y})=D \phi(\bar{y}), D^{2} \psi(\bar{y})=D^{2} \phi(\bar{y})$, this yields

$$
E_{*}\left(\bar{y}, u^{*}(\bar{y}), D \phi(\bar{y}), D^{2} \phi(\bar{y})\right) \leq 0,
$$

which implies that $u$ is a subsolution. q.e.d.

The above proof is due to Ishii [16], where he used $|y-\bar{y}|^{2}$ instead of $|y-\bar{y}|^{4}$. The modification term $|y-\bar{y}|^{4}$ is convenient to study the second order equations. The proof of Proposition 2.3 presented below is based on Perron's method and is essentially found in [16]. We begin with

Lemma 2.5. Suppose that $E$ is degenerate elliptic. Let $g: A \rightarrow \mathbb{R}$ be a supersolution of (2.1). Let $S$ be the collection of all subsolutions $v$ of (2.1), satisfying $v \leq g$ in $A$. If $v \in S$ is not a supersolution of (2.1), then there is a function $w \in S$ and a point $z \in A$ such that $v(z)<w(z)$.

Proof. Since $v$ is not a supersolution, there exist $\phi \in C^{2}(A)$ and $\bar{y} \in A$ such that

$$
\min _{A}\left(v_{*}-\phi\right)=\left(v_{*}-\phi\right)(\bar{y})=0
$$

and

$$
E^{*}\left(\bar{y}, v_{*}(\bar{y}), D \phi(\bar{y}), D^{2} \phi(\bar{y})\right)=E^{*}\left(\bar{y}, \phi(\bar{y}), D \phi(\bar{y}), D^{2} \phi(\bar{y})\right)<0 .
$$

We may assume here

$$
\left(v_{*}-\phi\right)(y) \geq|y-\bar{y}|^{4} \quad \text { for } y \in A,
$$

because the function $\phi$ can be modified as $\phi+|y-\bar{y}|^{4}$ if necessary. Evidently, we have $v_{*} \leq g_{*}$ in $A$. We now obtain $v_{*}(\bar{y})=\phi(\bar{y})<g_{*}(\bar{y})$, since, otherwise, it would contradict the fact that $g$ is a supersolution of (2.1). Considering that $E^{*}$ is u.s.c. and $\phi \in C^{2}(A)$, for sufficiently small $\delta>0$ we have

$$
\begin{gathered}
E^{*}\left(y, \phi(y)+\delta^{4} / 2, D \phi(y), D^{2} \phi(y)\right) \leq 0, \\
\phi(y)+\delta^{4} / 2 \leq g_{*}(y)
\end{gathered}
$$

for $y \in B_{2 \delta} \equiv B \cap \overline{B(\bar{y}, 2 \delta)}$, where $B$ is a compact neighborhood of $\bar{y}$, and $B(y, R)$ is the open ball of radius $R$ centered at $y$. (Such $B$ exists since $A$ is locally compact.) Since $E$ is degenerate elliptic, the 
inequality (2.5) indicates that, by $\phi \in C^{2}(A)$, the function $\phi(y)+\delta^{4} / 2$ is a subsolution in $B_{2 \delta}$. Furthermore, by (2.4) we have

$$
v(y) \geq v_{*}(y)-\delta^{4} / 2 \geq \phi(y)+\delta^{4} / 2 \text { on } B_{2 \delta} \backslash B_{\delta} .
$$

We now define $w(y)$ by

$$
w(y)= \begin{cases}\max \left\{\phi(y)+\delta^{4} / 2, v(y)\right\}, & y \in B_{\delta}, \\ v(y), & y \in A \backslash B_{\delta} .\end{cases}
$$

It follows from (2.7) that

$$
w(y)=\max \left\{\phi(y)+\delta^{4} / 2, v(y)\right\} \text { for } y \in B_{2 \delta} .
$$

According to Proposition 2.2, $w$ is a subsolution of $(2.1)$ over the whole of $A$ and, thus, $w \in S$ since (2.6) holds.

However, we have

$$
0=\left(v_{*}-\phi\right)(\bar{y})=\liminf _{t \downarrow 0}\{(v-\phi)(y) ; y \in A \text { and }|y-\bar{y}| \leq t\} .
$$

This implies that there is a point $z \in B_{\delta}$ such that $v(z)-\phi(z)<\delta^{4} / 2$, which yields $v(z)<w(z)$.

Proof of Proposition 2.3. We appeal to Perron's method. As in Lemma 2.5, we set $S=\{v ; v$ is a subsolution of (2.1) and $v \leq g\}$. Since $f \in S$, we see $S \neq \varnothing$. We define

$$
u(y)=\sup \{v(y) ; v \in S\} .
$$

By Proposition 2.2, $u$ is a subsolution of (2.1), so $u \in S$ since $u \leq g$. Suppose that $u$ were not a supersolution of (2.1). Then by Lemma 2.5 there would exist $w \in S$ such that $u(z)<w(z)$ for some $z \in A$. This is contrary to the definition of $u$. We thus conclude that $u$ is a solution of (2.1) and $f \leq u \leq g$.

Proof of Proposition 2.4. Let $\phi \in C^{2}(A)$ and $\bar{y} \in A$ satisfy

$$
\max _{A}\left(u^{*}-\phi\right)=\left(u^{*}-\phi\right)(\bar{y}) \text {. }
$$

Since $A$ is locally compact and $u_{k}^{*}<\infty$, there is a compact neighborhood $B$ of $\bar{y}$ on which $u_{k}$ is bounded from above. Since $u_{k}^{*}$ is u.s.c., one finds $y_{k} \in B(k=1,2, \cdots)$ such that

$$
\max _{B}\left(u_{k}^{*}-\phi\right)=\left(u_{k}^{*}-\phi\right)\left(y_{k}\right) \text {. }
$$

Here, we may assume $y_{k} \rightarrow \bar{y}(k \rightarrow \infty)$ because $u_{k} \rightarrow u(k \rightarrow \infty)$ uniformly on $B$. 
Since $\lim _{* \rightarrow \infty} E_{k} \geq E_{*}$ implies $\lim _{* k \rightarrow \infty} E_{k *} \geq E_{*}$, we have

$$
\begin{aligned}
& E_{*}\left(\bar{y}, u^{*}(\bar{y}), D \phi(\bar{y}), D^{2} \phi(\bar{y})\right) \\
& \leq \lim _{k \rightarrow \infty} E_{k *}\left(\bar{y}, u^{*}(\bar{y}), D \phi(\bar{y}), D^{2} \phi(\bar{y})\right) \\
& =\lim _{\substack{k \rightarrow \infty \\
\varepsilon \downarrow 0}} \inf _{\substack { l \geq k \\
\mid \begin{subarray}{c}{|z-\bar{z}|<\varepsilon \\
z \in J(A){ l \geq k \\
| \begin{subarray} { c } { | z - \overline { z } | < \varepsilon \\
z \in J ( A ) } }\end{subarray}} E_{l *}(y, s, q, Y),
\end{aligned}
$$

where $z=(y, s, q, Y)$ and $\bar{z}=\left(\bar{y}, u^{*}(\bar{y}), D \phi(\bar{y}), D^{2} \phi(\bar{y})\right)$. Since $u_{k}$ converges to $u$ uniformly on $B$, for each $\varepsilon>0$ we see $\left|z_{l}-\bar{z}\right|<\varepsilon$ with $z_{l}=\left(y_{l}, u_{l}^{*}\left(y_{l}\right), D \phi\left(y_{l}\right), D^{2} \phi\left(y_{l}\right)\right)$ for sufficiently large $l$. Hence, the right-hand side of $(2.8)$ is dominated by

$$
\liminf _{k \rightarrow \infty} E_{k *}\left(y_{k}, u_{k}^{*}\left(y_{k}\right), D \phi\left(y_{k}\right), D^{2} \phi\left(y_{k}\right)\right),
$$

so we obtain the desired inequality

$$
E_{*}\left(\bar{y}, u^{*}(\bar{y}), D \phi(\bar{y}), D^{2} \phi(\bar{y})\right) \leq 0
$$

which shows that $u$ is a subsolution of (2.1), once we prove

$$
E_{k *}\left(y_{k}, u_{k}^{*}\left(y_{k}\right), D \phi\left(y_{k}\right), D^{2} \phi\left(y_{k}\right)\right) \leq 0 \text { for all } k \geq 1 \text {. }
$$

Since $u_{k}$ is a subsolution of $E_{k}\left(y, u_{k}, D u_{k}, D^{2} u_{k}\right)=0$ in $A$ and $\max _{B}\left(u_{k}^{*}-\phi\right)-\left(u_{k}^{*}-\phi\right)\left(y_{k}\right)$, we have indeed (2.9) so the proof is now complete.

\section{Ishii's lemma on evolution equations}

We are concerned with a special form of (2.1) called the evolution equation,

$$
u_{t}+F\left(t, x, u, \nabla u, \nabla^{2} u\right)=0,
$$

where $\nabla$ stands for spatial derivatives. Our goal in this section is to prove a key lemma for our comparison theorem for (3.1) in $\S 4$. A similar result is first proved by Ishii [17] for (2.1) of nonevolution type. We state our main lemma.

Lemma 3.1. Let $\Omega$ be a domain in $\mathbb{R}^{n}$ and $T>0$. Let $u$ be a locally bounded upper semicontinuous (u.s.c.) subsolution of

$$
u_{t}+F\left(t, x, u, \nabla u, \nabla^{2} u\right)=0 \quad \text { in } \Omega_{T}=(0, T] \times \Omega,
$$

and let $v$ be a locally bounded lower semicontinuous (l.s.c.) supersolution of

$$
v_{t}+G\left(t, x, v, \nabla v, \nabla^{2} v\right)=0 \text { in } \Omega_{T},
$$


where $F, G: J=\Omega_{T} \times \mathbb{R} \times \mathbb{R}^{n} \times S^{n \times n} \rightarrow \widetilde{\mathbb{R}}$ are l.s.c. and u.s.c., respectively, and $r \mapsto F(t, x, r, p, X)$ and $r \mapsto G(t, x, r, p, X)$ are nondecreasing for all $(t, x, r, p, X) \in J$. Let $\phi \in C^{2}((0, T] \times \Omega \times \Omega)$ and $U$ be $a$ subdomain of $\Omega \times \Omega$. Let $(\bar{t}, \bar{x}, \bar{y}) \in U_{T}$ be a point such that

$$
u(\bar{t}, \bar{x})-v(\bar{t}, \bar{y})-\phi(\bar{t}, \bar{x}, \bar{y})=\sup _{U_{T}}(u-v-\phi),
$$

where $U_{T}=(0, T] \times U$. Suppose that

$$
u(t, \cdot) \text { and } v(t, \cdot) \text { are, for each } t \in[0, T], \text { Lipschitz con- }
$$
tinuous on $\bar{\Omega}$ and there is a constant $C>0$, independent of the time variable $t$, such that $\nabla_{x}^{2} u(t, x),-\nabla_{y}^{2} v(t, y) \geq$ $-C I$ in $\Omega_{T}$ (in the sense of distributions).

Then there are $X, Y \in S^{n \times n}$ such that

$$
\begin{gathered}
-C I \leq\left(\begin{array}{ll}
X & O \\
O & Y
\end{array}\right) \leq \nabla_{x, y}^{2} \phi(\bar{t}, \bar{x}, \bar{y}), \\
\frac{\partial \phi}{\partial t}(\bar{t}, \bar{x}, \bar{y})+F\left(\bar{t}, \bar{x}, u(\bar{t}, \bar{x}), \nabla_{x} \phi(\bar{t}, \bar{x}, \bar{y}), X\right) \\
-G\left(\bar{t}, \bar{y}, v(\bar{t}, \bar{y}),-\nabla_{y} \phi(\bar{t}, \bar{x}, \bar{y}),-Y\right) \leq 0,
\end{gathered}
$$

where I is the identity matrix.

We first prove a weaker version of Lemma 3.1 which is essentially Proposition IV.1 of [18].

Proposition 3.2. Suppose that $u, v, F$, and $G$ are as in Lemma 3.1. If the maximum point $(\bar{t}, \bar{x}, \bar{y})$ is in int $U_{T}=(0, T) \times U$, then the conclusion of Lemma 3.1 holds.

The idea of proof is given in [18]. However the proof is not explicitly stated. We give it here for completeness and the reader's convenience. The crucial tool is Jensen's lemma on semiconvex functions.

Lemma 3.3 [19, Lemma 3.10]. Let $D$ be a bounded domain in $\mathbb{R}^{d}$ and let $w$ be a Lipschitz continuous function in $\bar{D}$. For $\delta>0$, define

$$
\begin{aligned}
& \mathscr{G}_{\delta}=\left\{z \in D ; \text { for some } p \in \mathbb{R}^{d} \text { with }|p| \leq \delta,\right. \\
& \qquad w(\zeta) \leq w(z)+p \cdot(\zeta-z) \text { for all } \zeta \in D\} .
\end{aligned}
$$

Assume that there is a constant $K_{0}>0$ such that $D^{2} w \geq-K_{0} I$ in $D$ (in the sense of distributions). If $w$ has an interior maximum $\left(>\max _{\partial D} w\right)$, then there are constants $C_{0}>0$ and $\delta_{0}>0$ such that meas $\left(\mathscr{G}_{\delta}\right) \geq C_{0} \delta^{d}$ for all $\delta<\delta_{0}$. 
As is observed in [17, Lemma 5.2], Lemma 3.3 yields the following result since a convex function is almost everywhere differentiable by Alexandroff's theorem [2].

Lemma 3.4. Suppose that $w$ is as in Lemma 3.3. Let $\bar{z} \in D$ be a maximum point of $w$. Then there are sequences $\left\{z_{k}\right\} \subset D(k=1,2, \cdots)$ satisfying $z_{k} \rightarrow \bar{z}$ as $k \rightarrow \infty$ and $\left\{p_{k}\right\} \subset \mathbb{R}^{d}$ satisfying $\left|p_{k}\right| \leq 1 / k$ for all $k$ such that the functions $z \mapsto w(z)+p_{k} \cdot z$ attain a maximum at $z_{k}$ and have the second differential at $z_{k}$.

To prove Proposition 3.2 we approximate $u$ and $v$ by its sup and inf convolutions as in [18], [20]. We recall properties of these convolutions.

Lemma $3.5[21]$. Let $D$ be a bounded domain in $\mathbb{R}^{N}$ and $f, g: D \rightarrow \mathbb{R}$ be bounded u.s.c. and l.s.c. functions, respectively. For $\varepsilon>0$, we define

$$
\begin{array}{ll}
f^{\varepsilon}(y)=\sup _{z \in D}\left\{f(z)-\varepsilon^{-1}|y-z|^{2}\right\} & \text { for } y \in \bar{D}, \\
g_{\varepsilon}(y)=\inf _{z \in D}\left\{g(z)+\varepsilon^{-1}|y-z|^{2}\right\} & \text { for } y \in \bar{D},
\end{array}
$$

and call $f^{\varepsilon}, g_{\varepsilon}$ sup and inf convolutions, respectively. Then $f^{\varepsilon}\left(g_{\varepsilon}\right.$ resp.) is Lipschitz continuous on $\bar{D}$ and semiconvex (semiconcave resp.) on $D$. More precisely, $f^{\varepsilon}(y)+\varepsilon^{-1}|y|^{2}$ is convex on $D\left(g_{\varepsilon}(y)-\varepsilon^{-1}|y|^{2}\right.$ is concave on $D$ resp.). In the definitions of sup (inf resp.) convolution the $z$ 's may be restricted by $|y-z| \leq \lambda_{0} \sqrt{\varepsilon}$, where $\lambda_{0}=\left(2 \sup _{D}|f|\right)^{1 / 2}\left(\lambda_{0}=\left(2 \sup _{D}|g|\right)^{1 / 2}\right.$ resp.).

Lemma 3.6 [18], [20]. Suppose that $f, g$, and $D$ are as in Lemma 3.5. Let $E: D \times \mathbb{R} \times \mathbb{R}^{N} \times S^{N \times N} \rightarrow \widetilde{\mathbb{R}}$ be an l.s.c. (u.s.c. resp.) function and $s \mapsto E(y, s, q, Y)$ be nondecreasing. If $f$ ( $g$ resp.) is a sub- (superresp.) solution of

$$
E\left(y, u, D u, D^{2} u\right)=0 \text { in } D,
$$

then $f^{\varepsilon}\left(g_{\varepsilon}\right.$ resp.) is a sub- (super-resp.) solution of

$$
\begin{array}{ll}
E_{\varepsilon}\left(y, u, D u, D^{2} u\right)=0 & \text { in } D^{\varepsilon} \\
E^{\varepsilon}\left(y, u, D u, D^{2} u\right)=0 & \text { in } D^{\varepsilon} \text { resp. }
\end{array}
$$

Here $D^{\varepsilon}=\left\{y \in D ; \mathrm{d}(y, \partial D)>\lambda_{0} \sqrt{\varepsilon}\right\}$ and

$$
\begin{aligned}
& E_{\varepsilon}(y, s, q, Y)=\min \left\{E(z, s, q, Y) ;|y-z| \leq \lambda_{0} \sqrt{\varepsilon}, z \in D\right\} \\
& E^{\varepsilon}(y, s, q, Y)=\max \left\{E(z, s, q, Y) ;|y-z| \leq \lambda_{0} \sqrt{\varepsilon}, z \in D\right\} \text { resp. },
\end{aligned}
$$

where $\mathrm{d}(y, \partial D)$ denotes the distance between $y$ and $\partial D$.

These lemmas follow directly from definitions. 


\section{Proof of Proposition 3.2. Replacing $\phi$ by}

$$
(t, x, y) \mapsto \phi(t, x, y)+|t-\bar{t}|^{2}+|x-\bar{x}|^{4}+|y-\bar{y}|^{4}
$$

if necessary, we may assume that $u(t, x)-v(t, y)-\phi(t, x, y)$ attains a strict maximum over $\overline{U_{T}}$ at $(\bar{t}, \bar{x}, \bar{y})$. Hence, we may assume that $U_{T}$ is bounded and $u, v$ are bounded there since they are locally bounded.

Let $u^{\varepsilon}$ and $v_{\varepsilon}$ be, respectively, sup and inf convolutions in time of $u$ and $v$, i.e.

$$
\begin{aligned}
& u^{\varepsilon}(t, x)=\sup _{s \in[0, T]}\left\{u(s, x)-\varepsilon^{-1}(t-s)^{2}\right\}, \\
& v_{\varepsilon}(t, y)=\inf _{s \in[0, T]}\left\{v(s, y)+\varepsilon^{-1}(t-s)^{2}\right\} .
\end{aligned}
$$

Since $u^{\varepsilon}-v_{\varepsilon} \downarrow u-v$ in $\bar{U}_{T}$, a similar argument to [17, p. 29, 2nd paragraph; see also Proof of Theorem 4.1 in this paper] implies that $u^{\varepsilon}(t, x)-v_{\varepsilon}(t, y)-\phi(t, x, y)$ also has an interior (strict) maximum over $\overline{U_{T}}$ if $\varepsilon>0$ is sufficiently small and that the maximum point $\left(t^{\varepsilon}, x^{\varepsilon}, y^{\varepsilon}\right)$ tends to $(\bar{t}, \bar{x}, \bar{y})$ as $\varepsilon \downarrow 0$. By Lemma 3.5 we see $u^{\varepsilon}$ and $v_{\varepsilon}$ are Lipschitz continuous on $\overline{\Omega_{T}}$ and

$$
D_{t, x}^{2} u^{\varepsilon}(t, x),-D_{t, y}^{2} v_{\varepsilon}(t, y) \geq-\left(C+2 \varepsilon^{-1}\right) I \quad \text { in int } \Omega_{T}
$$

in the sense of distributions. We now apply Lemma 3.4 and observe that there are $\left(t_{k}^{\varepsilon}, x_{k}^{\varepsilon}, y_{k}^{\varepsilon}\right) \in \operatorname{int} U_{T}$ satisfying $\left(t_{k}^{\varepsilon}, x_{k}^{\varepsilon}, y_{k}^{\varepsilon}\right) \rightarrow\left(t^{\varepsilon}, x^{\varepsilon}, y^{\varepsilon}\right)$ as $k \rightarrow \infty$ and $\left(l_{k}^{\varepsilon}, p_{k}^{\varepsilon}, q_{k}^{\varepsilon}\right) \in \mathbb{R}^{2 n+1}$ satisfying $\left|l_{k}^{\varepsilon}\right|+\left|p_{k}^{\varepsilon}\right|+\left|q_{k}^{\varepsilon}\right| \leq 1 / k$ such that

$$
(t, x, y) \mapsto u^{\varepsilon}(t, x)-v_{\varepsilon}(t, y)-\phi(t, x, y)-l_{k}^{\varepsilon} t-p_{k}^{\varepsilon} \cdot x+q_{k}^{\varepsilon} \cdot y
$$

attains a maximum at $\left(t_{k}^{\varepsilon}, x_{k}^{\varepsilon}, y_{k}^{\varepsilon}\right)$ and has second differential at $\left(t_{k}^{\varepsilon}, x_{k}^{\varepsilon}, y_{k}^{\varepsilon}\right)$. These yield

$$
\begin{gathered}
D_{t, x, y}\left(u^{\varepsilon}-v_{\varepsilon}-\phi\right)\left(t_{k}^{\varepsilon}, x_{k}^{\varepsilon}, y_{k}^{\varepsilon}\right)=\left(l_{k}^{\varepsilon}, p_{k}^{\varepsilon},-q_{k}^{\varepsilon}\right), \\
\nabla_{x, y}^{2}\left(u^{\varepsilon}-v_{\varepsilon}-\phi\right)\left(t_{k}^{\varepsilon}, x_{k}^{\varepsilon}, y_{k}^{\varepsilon}\right) \leq 0,
\end{gathered}
$$

and by Lemma 3.6

$$
\begin{aligned}
& \frac{\partial u^{\varepsilon}}{\partial t}\left(t_{k}^{\varepsilon}, x_{k}^{\varepsilon}\right)+F_{\varepsilon}\left(t_{k}^{\varepsilon}, x_{k}^{\varepsilon}, u^{\varepsilon}\left(t_{k}^{\varepsilon}, x_{k}^{\varepsilon}\right), \nabla_{x} u^{\varepsilon}\left(t_{k}^{\varepsilon}, x_{k}^{\varepsilon}\right), \nabla_{x}^{2} u^{\varepsilon}\left(t_{k}^{\varepsilon}, x_{k}^{\varepsilon}\right)\right) \leq 0, \\
& \frac{\partial v_{\varepsilon}}{\partial t}\left(t_{k}^{\varepsilon}, y_{k}^{\varepsilon}\right)+G^{\varepsilon}\left(t_{k}^{\varepsilon}, y_{k}^{\varepsilon}, v_{\varepsilon}\left(t_{k}^{\varepsilon}, y_{k}^{\varepsilon}\right), \nabla_{y} v_{\varepsilon}\left(t_{k}^{\varepsilon}, y_{k}^{\varepsilon}\right), \nabla_{y}^{2} v_{\varepsilon}\left(t_{k}^{\varepsilon}, y_{k}^{\varepsilon}\right)\right) \geq 0 .
\end{aligned}
$$


Setting $X_{k}^{\varepsilon}=\nabla_{x}^{2} u^{\varepsilon}\left(t_{k}^{\varepsilon}, x_{k}^{\varepsilon}\right)$ and $Y_{k}^{\varepsilon}=-\nabla_{y}^{2} v_{\varepsilon}\left(t_{k}^{\varepsilon}, y_{k}^{\varepsilon}\right)$, we have

$$
-C I \leq\left(\begin{array}{cc}
X_{k}^{\varepsilon} & O \\
O & Y_{k}^{\varepsilon}
\end{array}\right) \leq \nabla_{x, y}^{2} \phi\left(t_{k}^{\varepsilon}, x_{k}^{\varepsilon}, y_{k}^{\varepsilon}\right) \text {, }
$$

$$
\begin{aligned}
& \frac{\partial \phi}{\partial t}\left(t_{k}^{\varepsilon}, x_{k}^{\varepsilon}, y_{k}^{\varepsilon}\right)+l_{k}^{\varepsilon} \\
& \quad+F_{\varepsilon}\left(t_{k}^{\varepsilon}, x_{k}^{\varepsilon}, u^{\varepsilon}\left(t_{k}^{\varepsilon}, x_{k}^{\varepsilon}\right), \nabla_{x} \phi\left(t_{k}^{\varepsilon}, x_{k}^{\varepsilon}, y_{k}^{\varepsilon}\right)+p_{k}^{\varepsilon}, X_{k}^{\varepsilon}\right) \\
& \quad-G^{\varepsilon}\left(t_{k}^{\varepsilon}, y_{k}^{\varepsilon}, v_{\varepsilon}\left(t_{k}^{\varepsilon}, y_{k}^{\varepsilon}\right),-\nabla_{y} \phi\left(t_{k}^{\varepsilon}, x_{k}^{\varepsilon}, y_{k}^{\varepsilon}\right)+q_{k}^{\varepsilon},-Y_{k}^{\varepsilon}\right) \leq 0 .
\end{aligned}
$$

Since $\nabla_{x, y}^{2} \phi\left(t_{k}^{\varepsilon}, x_{k}^{\varepsilon}, y_{k}^{\varepsilon}\right)$ is bounded from above uniformly in $\varepsilon$ and $k$, by compactness (see Lemma 5.3 in [17]) (3.5a) implies that there is an increasing sequence $\left\{k_{j}\right\}$, a decreasing sequence $\left\{\varepsilon_{j}\right\}$, and $X, Y \in S^{n \times n}$ such that $X_{j}=X_{k_{j}}^{\varepsilon_{j}} \rightarrow X$ and $Y_{j}=Y_{k_{j}}^{\varepsilon_{j}} \rightarrow Y$ as $j \rightarrow \infty$.

Let $s_{k}^{\varepsilon}$ be a maximum point of $u\left(s, x_{k}^{\varepsilon}\right)-\left(t_{k}^{\varepsilon}-s\right)^{2} / \varepsilon$ over $[0, T]$. We observe that $\left|\bar{t}-s_{k}^{\varepsilon}\right| \leq\left|\bar{t}-t_{k}^{\varepsilon}\right|+\lambda_{0} \sqrt{\varepsilon} \rightarrow 0$ as $\varepsilon \downarrow 0, k \rightarrow \infty$ and

$$
u^{\varepsilon}\left(t_{k}^{\varepsilon}, x_{k}^{\varepsilon}\right)=u\left(s_{k}^{\varepsilon}, x_{k}^{\varepsilon}\right)-\varepsilon^{-1}\left(t_{k}^{\varepsilon}-s_{k}^{\varepsilon}\right)^{2} \leq u\left(s_{k}^{\varepsilon}, x_{k}^{\varepsilon}\right),
$$

where $\lambda_{0}=\max \left((2 \sup |u|)^{1 / 2},(2 \sup |v|)^{1 / 2}\right)$. Since $u$ is u.s.c., we have

$$
\underset{\substack{\varepsilon \downarrow 0 \\ k \rightarrow \infty}}{\limsup } u^{\varepsilon}\left(t_{k}^{\varepsilon}, x_{k}^{\varepsilon}\right) \leq \underset{\substack{\varepsilon \downarrow 0 \\ k \rightarrow \infty}}{\limsup } u\left(s_{k}^{\varepsilon}, x_{k}^{\varepsilon}\right) \leq u(\bar{t}, \bar{x}) .
$$

By definition of $F_{\varepsilon}$ there is $\bar{s}_{k}^{\varepsilon} \in(0, T]$ satisfying $\left|t_{k}^{\varepsilon}-\bar{s}_{k}^{\varepsilon}\right| \leq \lambda_{0} \sqrt{\varepsilon}$ such that

$$
\begin{aligned}
& F_{\varepsilon}\left(t_{k}^{\varepsilon}, x_{k}^{\varepsilon}, u^{\varepsilon}\left(t_{k}^{\varepsilon}, x_{k}^{\varepsilon}\right), \nabla_{x} \phi\left(t_{k}^{\varepsilon}, x_{k}^{\varepsilon}, y_{k}^{\varepsilon}\right)+p_{k}^{\varepsilon}, X_{k}^{\varepsilon}\right) \\
& \quad=F\left(\bar{s}_{k}^{\varepsilon}, x_{k}^{\varepsilon}, u^{\varepsilon}\left(t_{k}^{\varepsilon}, x_{k}^{\varepsilon}\right), \nabla_{x} \phi\left(t_{k}^{\varepsilon}, x_{k}^{\varepsilon}, y_{k}^{\varepsilon}\right)+p_{k}^{\varepsilon}, X_{k}^{\varepsilon}\right) .
\end{aligned}
$$

Since $F$ is l.s.c. and $r \mapsto F(t, x, r, p, X)$ is nondecreasing, we now have

$$
\begin{aligned}
& \liminf _{j \rightarrow \infty} F\left(\bar{s}_{k}^{\varepsilon}, x_{k}^{\varepsilon}, u^{\varepsilon}\left(t_{k}^{\varepsilon}, x_{k}^{\varepsilon}\right), \nabla_{x} \phi\left(t_{k}^{\varepsilon}, x_{k}^{\varepsilon}, y_{k}^{\varepsilon}\right)+p_{k}^{\varepsilon}, X_{j}\right) \\
& \quad \geq F\left(\bar{t}, \bar{x}, u(\bar{t}, \bar{x}), \nabla_{x} \phi(\bar{t}, \bar{x}, \bar{y}), X\right), \quad \varepsilon=\varepsilon_{j}, \quad k=k_{j} .
\end{aligned}
$$

Similarly,

$$
\begin{aligned}
& \limsup _{j \rightarrow \infty} G^{\varepsilon}\left(t_{k}^{\varepsilon}, y_{k}^{\varepsilon}, v_{\varepsilon}\left(t_{k}^{\varepsilon}, y_{k}^{\varepsilon}\right),-\nabla_{y} \phi\left(t_{k}^{\varepsilon}, x_{k}^{\varepsilon}, y_{k}^{\varepsilon}\right)+q_{k}^{\varepsilon},-Y_{j}\right) \\
& \leq G\left(\bar{t}, \bar{y}, v(\bar{t}, \bar{y}),-\nabla_{y} \phi(\bar{t}, \bar{x}, \bar{y}),-Y\right), \quad \varepsilon=\varepsilon_{j}, \quad k=k_{j} .
\end{aligned}
$$

We now conclude from (3.5a) and (3.5b) that

$$
-C I \leq\left(\begin{array}{cc}
X & O \\
O & Y
\end{array}\right) \leq \nabla_{x, y}^{2} \phi(\bar{t}, \bar{x}, \bar{y})
$$


and

$$
\begin{gathered}
\frac{\partial \phi}{\partial t}(\bar{t}, \bar{x}, \bar{y})+F\left(\bar{t}, \bar{x}, u(\bar{t}, \bar{x}), \nabla_{x} \phi(\bar{t}, \bar{x}, \bar{y}), X\right) \\
-G\left(\bar{t}, \bar{y}, v(\bar{t}, \bar{y}),-\nabla_{y} \phi(\bar{t}, \bar{x}, \bar{y}),-Y\right) \leq 0,
\end{gathered}
$$

which is the same as $(3.4 \mathrm{a})$ and $(3.4 \mathrm{~b})$.

Remark 3.7. In Lemmas 3.1 and 3.6 the assumption that $r \mapsto$ $F(t, x, r, p, X)$ is nondecreasing is for simplicity only. Assertions in both Lemmas 3.1 and 3.6 hold without this assumption.

Proof of Lemma 3.1. Again we may assume that

$$
\Phi(t, x, y)=u(t, x)-v(t, y)-\phi(t, x, y)
$$

attains its strict maximum over $\overline{U_{T}}$ at $(\bar{t}, \bar{x}, \bar{y})$. By Proposition 3.2 we may assume $\bar{t}=T$.

We first construct a "barrier" near $T$. Since $\Phi$ is u.s.c., there is a continuous nondecreasing function $m:[0, \infty) \rightarrow[0, \infty)$ with $m(0)=0$ such that

$$
\Phi(T, \bar{x}, \bar{y})-\Phi(\bar{t}, \bar{x}, \bar{y}) \leq m(T-t) \text { for all } t, 0<t \leq T .
$$

We take a continuous nondecreasing function $M:[0, \infty) \rightarrow[0, \infty)$ such that $M(0)=0$ and $m(\sigma)<M(\sigma)$ for $\sigma>0$ and that $M(\sigma)$ is $C^{2}$ for $\sigma>0$. We now set

$$
\begin{aligned}
& \phi_{\alpha}(t, x, y)=\phi(t, x, y)-\alpha M(T-t), \quad \text { for } 0 \leq \alpha \leq 1 . \\
& \Phi_{\alpha}(t, x, y)=u(t, x)-v(t, y)-\phi_{\alpha}(t, x, y),
\end{aligned}
$$

Since $\Phi_{\alpha}$ is u.s.c., the set $\Sigma_{\alpha}$ of all maximum points of $\Phi_{\alpha}$ over $U_{T}$ is closed. We set

$$
t_{\alpha}=\sup \left\{t ;(t, x, y) \in \Sigma_{\alpha} \text { for some }(x, y) \in U\right\} \text {. }
$$

Since $\Sigma_{\alpha}$ is closed, there is $\left(x_{\alpha}, y_{\alpha}\right) \in U$ such that $\left(t_{\alpha}, x_{\alpha}, y_{\alpha}\right) \in \Sigma_{\alpha}$. We set

$$
\Lambda=\left\{\alpha \in[0,1] ; t_{\alpha}<T\right\}
$$

and observe that $\Lambda$ is an open set in $[0,1]$. Since

$$
\begin{aligned}
\Phi_{1}(T, \bar{x}, \bar{y}) & =\Phi(T, \bar{x}, \bar{y})<\Phi(t, \bar{x}, \bar{y})+M(T-t) \\
& =\Phi_{1}(t, \bar{x}, \bar{y}) \text { for all } t<T
\end{aligned}
$$

by the definition of $M$, we see $1 \in \Lambda$. Since $\bar{t}=T$, we see $0 \notin \Lambda$ which implies $\Lambda \neq[0,1]$. Let $\widetilde{\beta}$ be a boundary point of $\Lambda^{c}=\left\{\beta \in[0,1] ; t_{\beta}=\right.$ $T\}$. If $t_{\alpha}=T$, then

$$
\Phi_{\alpha}(T, x, y)=\Phi(T, x, y)
$$


holds, so we have $\left(x_{\alpha}, y_{\alpha}\right)=(\bar{x}, \bar{y})$. We thus observe that there is a sequence $\left\{\alpha_{j}\right\}$ in $\Lambda$ such that $\alpha_{j} \rightarrow \widetilde{\beta}$ as $j \rightarrow \infty$ and

$$
\left(t_{\alpha_{j}}, x_{\alpha_{j}}, y_{\alpha_{j}}\right)=\left(t_{j}, x_{j}, y_{j}\right) \rightarrow(T, \bar{x}, \bar{y}) \text { as } j \rightarrow \infty \text {. }
$$

By $t_{j}<T$ and Proposition 3.2 there are $X_{j}, Y_{j} \in S^{n \times n}$ such that

$$
-C I \leq\left(\begin{array}{cc}
X_{j} & O \\
O & Y_{j}
\end{array}\right) \leq \nabla_{x, y}^{2} \phi_{\alpha_{j}}\left(t_{j}, x_{j}, y_{j}\right),
$$

$$
\begin{gathered}
\frac{\partial \phi_{\alpha_{j}}}{\partial t}\left(t_{j}, x_{j}, y_{j}\right)+F\left(t_{j}, x_{j}, u\left(t_{j}, x_{j}\right), \nabla_{x} \phi_{\alpha_{j}}\left(t_{j}, x_{j}, y_{j}\right), X_{j}\right) \\
\quad-G\left(t_{j}, y_{j}, v\left(t_{j}, y_{j}\right),-\nabla_{y} \phi_{\alpha_{j}}\left(t_{j}, x_{j}, y_{j}\right),-Y_{j}\right) \leq 0
\end{gathered}
$$

Since

$$
\nabla_{x, y}^{2} \phi_{\alpha_{j}}\left(t_{j}, x_{j}, y_{j}\right)=\nabla_{x, y}^{2} \phi\left(t_{j}, x_{j}, y_{j}\right)
$$

is bounded from above uniformly in $j$, (3.6a) implies there is a sequence (still denoted $X_{j}, Y_{j}$ ) and $X, Y \in S^{n \times n}$ such that $X_{j} \rightarrow X$ and $Y_{j} \rightarrow Y$ as $j \rightarrow \infty$. We also see

$$
\frac{\partial \phi_{\alpha_{j}}}{\partial t}=\frac{\partial \phi}{\partial t}+\alpha M^{\prime}(T-t) \geq \frac{\partial \phi}{\partial t}
$$

since $\nabla \phi_{\alpha_{j}}=\nabla \phi$ and $M$ is nondecreasing. Since $F$ and $G$ are, respectively, l.s.c. and u.s.c., letting $j \rightarrow \infty$ in $(3.6 \mathrm{a}, \mathrm{b})$ yields $(3.4 \mathrm{a}, \mathrm{b})$ with $\bar{t}=T$ provided that

$$
\lim _{j \rightarrow \infty} u\left(t_{j}, x_{j}\right)=u(T, \bar{x}), \quad \lim _{j \rightarrow \infty} v\left(t_{j}, y_{j}\right)=v(T, \bar{y}) .
$$

It remains to prove (3.7). Suppose that (3.7) were false. Since $u$ and $v$ are u.s.c. and l.s.c., respectively, then either

$$
\liminf _{j \rightarrow \infty} u\left(t_{j}, x_{j}\right)<u(T, \bar{x}) \text { or } \limsup _{j \rightarrow \infty} v\left(t_{j}, y_{j}\right)>v(T, \bar{y})
$$

will hold. This would imply

$$
\liminf _{j \rightarrow \infty} \Phi_{\alpha_{j}}\left(t_{j}, x_{j}, y_{j}\right)<\Phi(T, \bar{x}, \bar{y}) .
$$

Since $\Phi_{\alpha_{j}}$ attains its maximum at $\left(t_{j}, x_{j}, y_{j}\right)$, we see

$$
\Phi_{\alpha_{j}}(T, \bar{x}, \bar{y})=\Phi(T, \bar{x}, \bar{y}) \leq \Phi_{\alpha_{j}}\left(t_{j}, x_{j}, y_{j}\right),
$$

which leads to a contradiction. 


\section{Parabolic comparison theorem}

We consider an evolution equation

$$
u_{t}+F\left(t, u, \nabla u, \nabla^{2} u\right)=0
$$

of degenerate parabolic type, i.e., $F$ is degenerate elliptic. In other words

$$
F(t, r, p, X+Y) \leq F(t, r, p, X) \text { for all } Y \geq O, Y \in S^{n \times n}
$$

holds where $F$ is defined. Our goal in this section is to show a comparison theorem on viscosity solutions of (4.1) in a bounded domain, even if $F(t, r, p, X)$ may not be continuous at $p=0$. A comparison theorem on viscosity solutions was first proved by P. L. Lions [22] for some special degenerate elliptic equations. Later his results were extended in [19], [17] for general degenerate elliptic equations $E\left(u(y), D u(y), D^{2} u(y)\right)=0$, where $E$ is assumed to be continuous in its variables (see also [6], [20]). A parabolic comparison theorem is also discussed in [24], [18], where $F=F(t, r, p, X)$ is still assumed to be continuous in $p$.

Let $\Omega$ be a bounded domain in $\mathbb{R}^{n}$ and $T>0$. We assume that $F$ satisfies

$$
\begin{gathered}
F: J_{0}=(0, T] \times \mathbb{R} \times\left(\mathbb{R}^{n} \backslash\{0\}\right) \times S^{n \times n} \rightarrow \mathbb{R} \text { is continuous, } \\
F \text { is degenerate elliptic on } J_{0},
\end{gathered}
$$

for all $M>0$, there is a constant $c_{0}=c_{0}(n, T, M)$ such that $r \mapsto F(t, r, p, X)+c_{0} r$ is nondecreasing for all $(t, r, p, X) \in J_{0}$ with $|r| \leq M$,

$$
-\infty<F_{*}(t, r, 0, O)=F^{*}(t, r, 0, O)<\infty
$$

for all $t \in[0, T], r \in \mathbb{R}$.

We now state our main comparison result.

Theorem 4.1. Let $u$ and $v$ be, respectively, sub- and supersolutions of (4.1) in $\Omega_{T}$. Suppose that $F$ satisfies (4.2a)-(4.2d). If $u^{*} \leq v_{*}$ on $\partial_{p} \Omega_{T}=\{0\} \times \Omega \cup[0, T] \times \partial \Omega$, then $u^{*} \leq v_{*}$ on $\Omega_{T}$.

Remark 4.2. We may assume that (4.1) has a form

$$
u_{t}+u+F\left(t, u, \nabla u, \nabla^{2} u\right)=0
$$

with

$\left(4.2 c^{\prime}\right) \quad r \mapsto F(t, r, p, X)$ is nondecreasing for all $(t, r, p, X) \in J_{0}$

if we replace $u$ ( $v$ resp.) by $e^{\lambda t} u\left(e^{\lambda t} v\right.$ resp.) with sufficiently large $\lambda$. 
The crucial step to prove Theorem 4.1 is the following property on viscosity solutions.

Lemma 4.3. Let $u$ and $v$ be, respectively, u.s.c. sub- and l.s.c. supersolutions of (4.3) in $\Omega_{T}$, where $F$ satisfies (4.2a), $(4.2 \mathrm{~b}),\left(4.2 \mathrm{c}^{\prime}\right)$, and (4.2d). Assume that $u$ and $v$ are locally bounded in $\Omega_{T}$. Let $\phi \in C^{2}\left(\mathbb{R}^{n}\right)$ such that

$$
\begin{gathered}
\nabla \phi(x)=0 \text { if and only if } x=0, \\
\phi(x)=\phi(-x)
\end{gathered}
$$

there is a constant $C_{1}>0$ such that $|\phi(x)-\phi(0)| \leq C_{1}|x|^{2}$ for sufficiently small $|x|$

(this actually follows from (4.4a) and $\left.\phi \in C^{2}\left(\mathbb{R}^{n}\right)\right)$,

$$
\phi(0) \leq \phi(x) \text { for all } x \in \mathbb{R}^{n} \text {. }
$$

Define $\psi(x, y)=\phi(x-y)$ and $Q_{T}=(0, T] \times \Omega \times \Omega$. Assume that

$u(t, \cdot)$ and $v(t, \cdot)$ are Lipschitz continuous on $\bar{\Omega}$ for each $t \in[0, T]$ and there is a constant $C_{2}>0$ (independent of $t$ ) such that $\nabla_{x}^{2} u(t, x),-\nabla_{y}^{2} v(t, y) \geq-C_{2} I$ on $\Omega_{T}$ (in the sense of distributions),

(4.5b) $(\bar{t}, \bar{x}, \bar{y}) \in Q_{T}$ is a point such that

$$
\begin{aligned}
u(\bar{t}, \bar{x})-v(\bar{t}, \bar{y})-\psi(\bar{x}, \bar{y}) & =\sup _{Q_{T}}(u-v-\psi) \\
& >\max _{\partial_{p} Q_{T}}(u-v-\psi),
\end{aligned}
$$

where $\partial_{p} Q_{T}=\{0\} \times \Omega \times \Omega \cup[0, T] \times \partial(\Omega \times \Omega)$. Then $u(\bar{t}, \bar{x})-v(\bar{t}, \bar{y}) \leq 0$.

Proof. We divide the proof into several cases depending on $\bar{x}$ and $\bar{y}$. We first discuss the case $\bar{x} \neq \bar{y}$.

Case $1(\bar{x} \neq \bar{y})$. Since $\bar{x} \neq \bar{y}$ implies $\nabla \phi(\bar{x}-\bar{y}) \neq 0$, the proof parallels the argument found in [17, pp. 29-30]. By Lemma 3.1 and $\partial \psi / \partial t=0$, there are $X, Y \in S^{n \times n}$ such that

$$
\begin{aligned}
-C_{2} I \leq\left(\begin{array}{ll}
X & O \\
O & Y
\end{array}\right) & \leq \nabla_{x, y}^{2} \psi(\bar{x}, \bar{y}) \\
& =\left(\begin{array}{cc}
\nabla^{2} \phi(\bar{x}-\bar{y}) & -\nabla^{2} \phi(\bar{x}-\bar{y}) \\
-\nabla^{2} \phi(\bar{x}-\bar{y}) & \nabla^{2} \phi(\bar{x}-\bar{y})
\end{array}\right),
\end{aligned}
$$




$$
\begin{aligned}
& u(\bar{t}, \bar{x})+F_{*}\left(\bar{t}, u(\bar{t}, \bar{x}), \nabla_{x} \psi(\bar{x}, \bar{y}), X\right) \\
& \quad-v(\bar{t}, \bar{y})-F^{*}\left(\bar{t}, v(\bar{t}, \bar{y}),-\nabla_{y} \psi(\bar{x}, \bar{y}),-Y\right) \leq 0 .
\end{aligned}
$$

The second inequality in (4.6a) is equivalent to

$$
\begin{aligned}
X p \cdot p+Y q \cdot q \leq & \nabla^{2} \phi(\bar{x}-\bar{y}) p \cdot p-2 \nabla^{2} \phi(\bar{x}-\bar{y}) p \cdot q \\
& +\nabla^{2} \phi(\bar{x}-\bar{y}) q \cdot q \text { for all } p, q \in \mathbb{R}^{n} .
\end{aligned}
$$

This yields $X \leq-Y$ by taking $p=q$. Since $F_{*}=F^{*}=F$ on $J_{0}$, and

$$
\nabla_{x} \psi(x, y)=-\nabla_{y} \psi(x, y)=\nabla \phi(x-y)
$$

and $\nabla \phi(\bar{x}-\bar{y}) \neq 0$, applying (4.2b) and (4.2c') in (4.6b) with $X \leq-Y$ we obtain

$$
u(\bar{t}, \bar{x})-v(\bar{t}, \bar{y}) \leq 0 .
$$

Case $2(\bar{x}=\bar{y})$. We set

$$
\widetilde{\psi}(t, x, y)=\psi(x, y)+(\bar{t}-t)^{2},
$$

and observe that $w-\widetilde{\psi}$ attains its maximum over $Q_{T}$ at $(\bar{t}, \bar{x}, \bar{x})$, where

$$
w(t, x, y)=u(t, x)-v(t, y) .
$$

For $\eta \in \mathbb{R}^{n}$ we set

$$
\Phi_{\eta}(t, x, y)=w(t, x, y)-\phi(x-y-\eta)-(\bar{t}-t)^{2}
$$

and divide the situation into the following Case $2 \mathrm{a}$ and its negation Case 2b.

Case 2a. For some $\kappa>0$ there is $\left(t_{\eta}, x_{\eta}, y_{\eta}\right) \in Q_{\bar{t}}$ with $x_{\eta}-y_{\eta}=\eta$ such that

$$
\begin{aligned}
\Phi_{\eta}\left(t_{\eta}, x_{\eta},\right. & \left.y_{\eta}\right) \\
& =\sup \left\{\Phi_{\eta}(t, x, y) ; x, y \in \Omega,|x-y|<\kappa, t \in(0, \bar{t}]\right\}
\end{aligned}
$$

for all $\eta \in \mathbb{R}^{n}$ with $|\eta|<\kappa$.

We discuss Case $2 \mathrm{a}$. We set

$$
f(\eta)=\sup \left\{w\left(t_{\eta}, x, y\right)-\left(\bar{t}-t_{\eta}\right)^{2} ; x, y \in \Omega, x-y=\eta\right\}
$$

By (4.7) we have

$$
w(t, x, y)-(\bar{t}-t)^{2} \leq \phi(x-y-\eta)+w\left(t_{\eta}, x_{\eta}, y_{\eta}\right)-\left(\bar{t}-t_{\eta}\right)^{2}-\phi(0)
$$

for all $x, y \in \Omega$ with $|x-y|<\kappa, t \in(0, T]$. This, in particular, yields

$$
f(\xi) \leq \phi(\xi-\eta)+f(\eta)-\phi(0)
$$


by taking $x-y=\xi$ and $t=t_{\xi}$. From (4.4b) it now follows that

$$
-\phi(\xi-\eta)+\phi(0) \leq f(\xi)-f(\eta) \leq \phi(\xi-\eta)-\phi(0)
$$

which together with (4.4c) implies that $f$ is differentiable in $\eta,|\eta|<\kappa$, and that $\nabla f$ identically equals zero. Hence, $f(\eta)$ is a constant for $|\eta|<$ $\kappa$. This implies

$$
\sup _{|x-y|<\kappa}\left\{w\left(t_{\eta}, x, y\right)-\left(\bar{t}-t_{\eta}\right)^{2}\right\}=\sup _{x \in \Omega} w(\bar{t}, x, x) .
$$

We shall show that

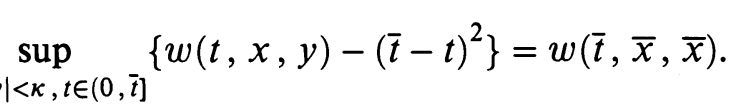

Property (4.7) with $\eta=0$ yields

$$
\sup _{|x-y|<\kappa, t \in(0, \bar{t}]}\left\{w(t, x, y)-(\bar{t}-t)^{2}-\phi(x-y)\right\}=w(\bar{t}, \bar{x}, \bar{x})-\phi(0),
$$

from which we deduce

$$
w(\bar{t}, \bar{x}, \bar{x})-\phi(0) \geq w(\bar{t}, x, x)-\phi(0) \text { for all } x \in \Omega .
$$

We thus conclude

$$
\sup _{x \in \Omega} w(\bar{t}, x, x)=w(\bar{t}, \bar{x}, \bar{x})
$$

Observe that

$$
\begin{array}{r}
\sup _{x-y=\eta, t \in(0, \bar{t}]}\left\{w(t, x, y)-(\bar{t}-t)^{2}\right\} \leq w\left(t_{\eta}, x_{\eta}, y_{\eta}\right)-\left(\bar{t}-t_{\eta}\right)^{2}, \\
|\eta|<\kappa,
\end{array}
$$

by taking $x-y=\eta$ in (4.7). Applying (4.8) and (4.10) to (4.11) yields

$$
\sup _{|x-y|<\kappa, t \in(0, \bar{t}]}\left\{w(t, x, y)-(\bar{t}-t)^{2}\right\} \leq w(\bar{t}, \bar{x}, \bar{x}) .
$$

The converse inequality is trivial, so we now obtain (4.9).

By (4.9) we now apply Lemma 3.1 with $\phi=(\bar{t}-t)^{2}$ and

$$
U=\{(x, y) \in \Omega \times \Omega ;|x-y|<\kappa\},
$$

and observe that there are $X, Y \in S^{n \times n}$ such that

$$
\begin{gathered}
\left(\begin{array}{ll}
X & O \\
O & Y
\end{array}\right) \leq O \\
u(\bar{t}, \bar{x})+F_{*}(\bar{t}, u(\bar{t}, \bar{x}), 0, X)-v(\bar{t}, \bar{x}) \\
-F^{*}(\bar{t}, v(\bar{t}, \bar{x}), 0,-Y) \leq 0 .
\end{gathered}
$$


The estimate (4.12a) yields $X \leq O \leq-Y$. By (4.2b) we now have

$$
F_{*}(t, r, 0, X) \geq F_{*}(t, r, 0, O), \quad F^{*}(t, r, 0,-Y) \leq F^{*}(t, r, 0, O) .
$$

Applying these with $\left(4.2 \mathrm{c}^{\prime}\right)$ and $(4.2 \mathrm{~d})$ to $(4.12 \mathrm{~b})$ gives

$$
u(\bar{t}, \bar{x})-v(\bar{t}, \bar{x}) \leq 0 \text {, }
$$

and the proof of Case $2 \mathrm{a}$ is now complete. The assumption (4.2d) is only invoked here.

It remains to discuss the following Case $2 \mathrm{~b}$. In this case there is a sequence $\left\{\eta_{i}\right\}$ in $\mathbb{R}^{n}$ with $\left|\eta_{i}\right|<1 / i$ and $\left(t_{i}, x_{i}, y_{i}\right) \in Q_{\bar{t}}$ with $x_{i}-y_{i} \neq$ $\eta_{i}(i=1,2, \ldots)$ such that $(4.7)$ holds. Since $\nabla \phi\left(x_{i}-y_{i}-\eta_{i}\right) \neq 0$, the same argument in Case 1 shows that

$$
u\left(t_{i}, x_{i}\right)-v\left(t_{i}, y_{i}\right) \leq 0 .
$$

There is a convergent subsequence (still denoted $\left\{\left(t_{i}, x_{i}, y_{i}\right)\right\}$ ) in $Q_{T}$. Since the maximum of $\Phi_{0}$ is not attained for $t<\bar{t}$, its limit is expressed as $(\bar{t}, \overline{\bar{x}}, \overline{\bar{y}}) \in Q_{T}$ by $(4.5 \mathrm{~b})$. The limit $(\bar{t}, \overline{\bar{x}}, \overline{\bar{y}})$ is a maximum point of $\Phi_{0}$ since $\Phi_{0}$ is u.s.c. Letting $i \rightarrow \infty$ in (4.13) yields

$$
u(\bar{t}, \overline{\bar{x}})-v(\bar{t}, \overline{\bar{y}}) \leq 0
$$

provided that

$$
\lim _{i \rightarrow \infty} u\left(t_{i}, x_{i}\right)=u(\bar{t}, \overline{\bar{x}}), \quad \lim _{i \rightarrow \infty} v\left(t_{i}, y_{i}\right)=v(\bar{t}, \overline{\bar{y}}) .
$$

By (4.4d) it follows from (4.14) that

$$
u(\bar{t}, \bar{x})-v(\bar{t}, \bar{x}) \leq 0 .
$$

It remains to prove (4.15) to complete the proof of Case 2. The idea is similar to the proof of (3.7). Suppose that (4.15) were false. Then we would have

$$
\liminf _{i \rightarrow \infty} \Phi_{\eta_{i}}\left(t_{i}, x_{i}, y_{i}\right)<\Phi_{0}(\bar{t}, \overline{\bar{x}}, \overline{\bar{y}}),
$$

since $u$ and $v$ are u.s.c. and l.s.c., respectively. However, since $\Phi_{\eta_{i}}$ attains its maximum at $\left(t_{i}, x_{i}, y_{i}\right)$ and $\Phi_{0}$ attains its maximum at $(\bar{t}, \bar{x}, \bar{x})$ and $(\bar{t}, \overline{\bar{x}}, \overline{\bar{y}})$, we see

$$
\begin{aligned}
\Phi_{\eta_{i}}\left(t_{i}, x_{i}, y_{i}\right) & \geq \Phi_{\eta_{i}}(\bar{t}, \bar{x}, \bar{y})=w(\bar{t}, \bar{x}, \bar{x})-\phi\left(\eta_{i}\right) \\
& \geq \Phi_{0}(\bar{t}, \overline{\bar{x}}, \overline{\bar{y}})-C_{1} / i^{2}
\end{aligned}
$$

by (4.4b) and (4.4c). This leads to a contradiction to (4.16), so we obtain (4.15). q.e.d. 
Lemma 4.3 plays a crucial role in the proof of Theorem 4.1. The remaining part of the proof of Theorem 4.1 parallels the argument in [17, pp. 29-30] as is described below.

Proof of Theorem 4.1. By Remark 4.2 we may assume that the equation has the form (4.3). By the definition of sub- (super- resp.) solution and the boundedness of $\Omega_{T}$, replacing $u$ ( $v$ resp.) by

$$
\{\max (u(t, x),-M)\}^{*} \quad\left(\{\min (v(t, x), M)\}_{*} \text { resp. }\right)
$$

for sufficiently large $M$ we may assume that $u$ ( $v$ resp.) is bounded u.s.c. (1.s.c. resp.) on $\overline{\Omega_{T}}=\Omega_{T} \cup \partial_{p} \Omega_{T}$. The assumption $u^{*} \leq v_{*}$ implies there is a modulus function $m$ (i.e., $m:[0, \infty) \rightarrow[0, \infty)$ is continuous, nondecreasing, concave and $m(0)=0)$ such that

$$
u(t, x)-v(t, y) \leq m(|x-y|) \text { on } \partial_{p} Q_{T},
$$

where

$$
Q_{T}=(0, T] \times \Omega \times \Omega \text { and } \partial_{p} Q_{T}=\{0\} \times \Omega \times \Omega \cup[0, T] \times \partial(\Omega \times \Omega) \text {. }
$$

We choose $\left\{a_{\lambda}\right\}_{\lambda \in A}$ and $\left\{b_{\lambda}\right\}_{\lambda \in A}$ as positive numbers, with $A$ a suitable index set, such that

$$
m(\sigma)=\inf _{\lambda \in A}\left(a_{\lambda} \sigma+b_{\lambda}\right)
$$

For fixed $\lambda \in A$ and $\delta>0$ define $\phi, \psi$ by

$$
\phi(z)=a_{\lambda}\left(|z|^{2}+\delta\right)^{1 / 2}+b_{\lambda} \text { and } \psi(x, y)=\phi(x-y) .
$$

We set $w(t, x, y)=u(t, x)-v(t, y)$ and want to prove $w \leq \psi$ on $Q_{T}$, which obviously ensures our assertion. To do this, we suppose that $\sup _{Q_{T}}(w-\psi)>0$, and then get a contradiction. Set

$$
w^{\varepsilon}(t, x, y)=u^{\varepsilon}(t, x)-v_{\varepsilon}(t, y) \text { for } \varepsilon>0,
$$

where $u^{\varepsilon}\left(v_{\varepsilon}\right.$ resp.) is the sup (inf resp.) convolution in $x$ (y resp.). We know that $w^{\varepsilon} \downarrow w$ on $\overline{Q_{T}}$ as $\varepsilon \downarrow 0$, and $w^{\varepsilon}$ and $w$ are u.s.c. on $\overline{Q_{T}}$. As $\varepsilon \downarrow 0$ we see

$$
\left\{\left(w^{\varepsilon}-\phi\right)(t, x, y)-\max _{\partial_{p} Q_{T}}(w-\phi)\right\}^{+} \downarrow 0 \text { for }(t, x, y) \in \partial_{p} Q_{T},
$$

where $f(z)^{+}=\max (f(z), 0)$. By Dini's theorem this convergence is uniform on $\partial_{p} Q_{T}$. Note also that $\max _{\partial_{p} Q_{T}}(w-\phi)<0<\sup _{Q_{T}}(w-\phi)$. Thus we find that $w^{\varepsilon}-\phi$ attains a maximum over $Q_{T}$ at a point $(\bar{t}, \bar{x}, \bar{y})$ of $Q_{T}^{\sigma}$ if $\varepsilon>0$ and $\sigma>0$ are sufficiently small, where $Q_{T}^{\sigma}=(\sigma, T] \times$ $\Omega^{\sigma} \times \Omega^{\sigma}$ and $\Omega^{\sigma}=\{x \in \Omega ; \mathrm{d}(x, \partial \Omega)>\sigma\}$. We get, for sufficiently small 
$\sigma>0$

$$
\left(w^{\varepsilon}-\psi\right)(\bar{t}, \bar{x}, \bar{y})=\sup _{Q_{T}^{\sigma}}\left(w^{\varepsilon}-\psi\right)>\max _{\partial_{p} Q_{T}^{\sigma}}\left(w^{\varepsilon}-\psi\right) .
$$

Since $u^{\varepsilon}\left(v_{\varepsilon}\right.$ resp.) is a sub- (super- resp.) solution of (4.3) in $\Omega_{T}^{\sigma}$ by Lemma 3.6, and since $u^{\varepsilon}(t, \cdot)\left(v_{\varepsilon}(t, \cdot)\right.$ resp.) by Lipschitz continuous on $\bar{\Omega}$ for each $t \in[0, T]$ and $\nabla_{x}^{2} u^{\varepsilon}(t, x),-\nabla_{y}^{2} v^{\varepsilon}(t, y) \geq-2 \varepsilon^{-1} I$ in $\Omega_{T}$ (in the sense of distributions)

by Lemma 3.5, applying Lemma 4.3 with $u^{\varepsilon}$ and $v_{\varepsilon}$ yields $w^{\varepsilon}(\bar{t}, \bar{x}, \bar{y}) \leq$ 0 . Hence, we have

$$
w^{\varepsilon}(t, x, y)-\psi(x, y) \leq 0 \text { in } Q_{T},
$$

which is a contradiction. Therefore, $\sup _{Q_{T}}(w-\phi) \leq 0$, i.e.,

$$
u(t, x)-v(t, y) \leq a_{\lambda}\left(|x-y|^{2}+\delta\right)^{1 / 2}+b_{\lambda} \text { on } Q_{T}
$$

Letting $\delta \rightarrow 0$ and taking the infimum for $\lambda \in A$, we find $u(t, x)-$ $v(t, y) \leq m(|x-y|)$ on $Q_{T}$ holds. We now conclude that $u \leq v$ on $\Omega_{T}$ and this completes the proof. q.e.d.

As in [17], Theorem 4.1 yields uniqueness and existence of solutions by Perron's method.

Theorem 4.4. Suppose $\Omega$ is a bounded domain in $\mathbb{R}^{n}$, and $F$ is as in Theorem 4.1. For given data $g \in C\left(\partial_{p} \Omega_{T}\right)$ there is at most one viscosity solution $u$ of the initial boundary value problem of (4.1) in $\Omega_{T}$, with $u^{*}=u_{*}=g$ on $\partial_{p} \Omega_{T}$.

This follows directly from Theorem 4.1 by comparison.

Theorem 4.5. Suppose $\Omega$ is a bounded domain in $\mathbb{R}^{n}$, and $F$ is as in Theorem 4.1. Suppose that there is a subsolution $f$ and a supersolution $g$ of (4.1) in $\Omega_{T}$ satisfying $f \leq g$ on $\Omega_{T}$ and $f_{*}=g^{*}$ on $\partial_{p} \Omega_{T}$. Then there is a viscosity solution $u$ of (4.1) satisfying $u \in C\left(\overline{\Omega_{T}}\right)$ and $f \leq u \leq g$ on $\overline{\Omega_{T}}$, where $\overline{\Omega_{T}}=\partial_{p} \Omega_{T} \cup \Omega_{T}$.

Proof. By Proposition 2.3 there is a viscosity solution $u$ of (4.1) in $\Omega_{T}$ satisfying $f \leq u \leq g$ on $\Omega_{T}$. Assumption $f_{*}=g^{*}$ on $\partial_{p} \Omega_{T}$ implies $u^{*} \leq u_{*}$ on $\partial_{p} \Omega_{T}$, which leads to $u^{*} \leq u_{*}$ on $\Omega_{T}$ by Theorem 4.1. Hence, we have $u^{*}=u_{*}$, i.e., $u \in C\left(\overline{\Omega_{T}}\right)$.

Remark 4.6. Our method also yields a comparison theorem for elliptic equations $u+F\left(u, \nabla u, \nabla^{2} u\right)=0$. In fact Theorem 3.1 in [17] can be extended even when $F$ is not continuous at $p=0$ provided (4.2d) holds. 


\section{Geometric parabolic equations}

In this section we prove that geometric, degenerate parabolic equations are invariant under an (orientation-preserving) change of a dependent variable even in the viscosity sense. Our main tools are an approximation of solutions by sup (inf) convolution in Lemma 3.5, Jensen's lemma (Lemmas 3.3 and 3.4), and stability (Proposition 2.4). We begin by discussing geometric equations of nonevolution type.

Definition 5.1. Let $E: W \rightarrow \mathbb{R}$, where $W \subset J\left(\mathbb{R}^{N}\right)=\mathbb{R}^{N} \times \mathbb{R} \times \mathbb{R}^{N} \times$ $S^{N \times N}$. We say the equation $E=0$ is geometric in $W$ if $E$ satisfies

$$
E=E(y, s, q, Y) \text { is independent of } s,
$$

and for $\lambda>0$ and $\mu \in \mathbb{R}$ there is $C_{i}=C_{i}(\lambda, \mu)>0(i=1,2)$ such that

$$
C_{1} E(y, q, Y) \leq E(y, \lambda q, \lambda Y+\mu q \otimes q) \leq C_{2} E(y, q, Y)
$$

holds whenever each term is well defined. Here $\otimes$ denotes a tensor product of vectors in $\mathbb{R}^{N}$. It is easy to see that the equations $E_{*}=0$ and $E^{*}=0$ are geometric in $\bar{W}$ if $E=0$ is geometric in $W$.

The following is our main result for geometric, degenerate elliptic equations in this section.

Theorem 5.2. Let $A$ be an open set in $\mathbb{R}^{N}$, and $W$ be a dense subset of $J(A)$. Let $u$ be a locally bounded viscosity sub-(super-resp.) solution of

$$
E\left(y, u, D u, D^{2} u\right)=0 \text { in } A .
$$

If $E$ is degenerate elliptic and $E=0$ is geometric, then $\theta(u)$ is a viscosity sub- (super-resp.) solution whenever $\theta: \mathbb{R} \rightarrow \mathbb{R}$ is a continuous nondecreasing function.

We first prove Theorem 5.2 assuming more regularity on $\theta$ and $u$ so that Jensen's lemma is applicable.

Lemma 5.3. Let $A, W, u$, and $E$ be as in Theorem 5.2. Suppose that $A$ is bounded and that $u$ is semiconvex (concave resp.) in $A$ and Lipschitz continuous on $\bar{A}$. If $\theta$ is an increasing function in $C^{2}(\mathbb{R})$ with $\theta^{\prime}>0$, then $\theta(u)$ is a sub- (super- resp.) solution of (5.2).

Proof. We only prove the case where $u$ is a subsolution since the other case can be proved similarly. Since $u$ is semiconvex in $A$ and Lipschitz continuous on a compact set $\bar{A}$, we see

$$
D^{2}(\theta \circ u)=\left(\theta^{\prime \prime} \circ u\right) D u \otimes D u+\left(\theta^{\prime} \circ u\right) D^{2} u \geq-C I \quad \text { in } A
$$

(in the sense of distribution) with some $C$ independent of $y$, where 
$(f \circ g)(y)=f(g(y))$. This implies that $\theta \circ u$ is also semiconvex in $A$. We also observe that $\theta \circ u$ is Lipschitz continuous in $\bar{A}$.

Suppose that $\phi \in C^{2}(A)$ and $\bar{y} \in A$ satisfy

$$
\max _{A}(\theta \circ u-\phi)=\theta(u(\bar{y}))-\phi(\bar{y}) .
$$

(Here we may assume $\phi \in C^{2}(\bar{A})$ by modifying $\phi$ away from $\bar{y}$.) Applying Lemma 3.4 to $\theta \circ u-\phi$, we see there are sequences $\left\{y_{k}\right\}$ in $A$ and $\left\{q_{k}\right\}$ in $\mathbb{R}^{N}$ such that

$$
\begin{gathered}
y_{k} \rightarrow \bar{y} \text { and } q_{k} \rightarrow 0 \text { as } k \rightarrow \infty, \\
\max _{A}\left(\theta \circ u-\phi-q_{k} \cdot y\right)=\theta\left(u\left(y_{k}\right)\right)-\phi\left(y_{k}\right)-q_{k} \cdot y_{k},
\end{gathered}
$$

$\theta \circ u$ has second differential at $y_{k}$.

Thus

$$
E_{*}\left(y_{k}, D(\theta \circ u)\left(y_{k}\right), D^{2}(\theta \circ u)\left(y_{k}\right)\right) \leq C_{2} E_{*}\left(y_{k}, D u\left(y_{k}\right), D^{2} u\left(y_{k}\right)\right)
$$

since $E_{*}=0$ is geometric in $J(A)$, and (5.5) implies

$$
\begin{aligned}
D(\theta \circ u)\left(y_{k}\right) & =\theta^{\prime}\left(u\left(y_{k}\right)\right) D u\left(y_{k}\right), \\
D^{2}(\theta \circ u)\left(y_{k}\right) & =\theta^{\prime \prime}\left(u\left(y_{k}\right)\right) D u\left(y_{k}\right) \otimes D u\left(y_{k}\right)+\theta^{\prime}\left(u\left(y_{k}\right)\right) D^{2} u\left(y_{k}\right)
\end{aligned}
$$

with $\theta^{\prime}\left(u\left(y_{k}\right)\right)>0$. (5.6) yields

$$
E_{*}\left(y_{k}, D(\theta \circ u)\left(y_{k}\right), D^{2}(\theta \circ u)\left(y_{k}\right)\right) \leq 0,
$$

since $u$ is a subsolution of (5.2) and the right-hand side of (5.6) is nonpositive; notice that $u(y)$ has second differential at $y=y_{k}$ by (5.5) since $\theta \in C^{2}(\mathbb{R})$ with $\theta^{\prime}>0$ implies that the inverse $\theta^{-1} \in C^{2}(\mathbb{R})$.

By (5.4) we have

$$
D(\theta \circ u)\left(y_{k}\right)=D \phi\left(y_{k}\right)+q_{k}, \quad D^{2}(\theta \circ u)\left(y_{k}\right) \leq D^{2} \phi\left(y_{k}\right) .
$$

Since $E_{*}$ is degenerate elliptic, (5.7) gives

$$
E_{*}\left(y_{k}, D \phi\left(y_{k}\right)+q_{k}, D^{2} \phi\left(y_{k}\right)\right) \leq 0 .
$$

Since $E_{*}$ is 1.s.c., letting $k \rightarrow \infty$ in (5.8) and noting (5.3), we obtain

$$
E_{*}\left(\bar{y}, D \phi(\bar{y}), D^{2} \phi(\bar{y})\right) \leq 0,
$$

which shows that $\theta \circ u=\theta(u)$ is a subsolution of (5.2) in $A$. q.e.d.

We need to approximate $\theta \in C(\mathbb{R})$ by $C^{2}$ functions. 
Lemma 5.4. Suppose that $\theta: \mathbb{R} \rightarrow \mathbb{R}$ is a continuous nondecreasing function. Then there is a sequence $\left\{\theta_{k}\right\}$ in $C^{2}(\mathbb{R})$ of increasing functions with $\theta_{k}^{\prime}>0$ such that $\theta_{k} \rightarrow \theta$ uniformly in $\mathbb{R}$ as $k \rightarrow \infty$.

Proof. $1^{\circ}$. We approximate $\theta$ by nondecreasing piecewise linear functions. For an integer $j$ and positive integer $k$ we set

$$
a_{j}^{(k)}=\sup \{t ; \theta(t) \leq j / k\} .
$$

Since $\theta$ is a continuous nondecreasing function, we see the sequence $\left\{a_{j}^{(k)}\right\}_{j=-\infty}^{\infty}$ in $\widetilde{\mathbb{R}}=\mathbb{R} \cup\{ \pm \infty\}$ has no accumulation points in $\mathbb{R}$ and $a_{j}^{(k)}<a_{j+1}^{(k)}$ (unless both are infinite with the same sign). We now define a continuous nondecreasing piecewise linear function $\theta_{k}$ such that it is linear except at $a_{j}^{(k)}$ and agrees with $\theta$ at $a_{j}^{(k)} \in \mathbb{R}$. It is easy to see that $\theta_{k} \rightarrow \theta$ uniformly in $\mathbb{R}$ as $k \rightarrow \infty$.

$2^{\circ}$. We approximate a nondecreasing piecewise linear function $\theta_{k}$ by a nondecreasing $C^{2}$ function. This is easy because all we need is to mollify $\theta_{k}$ near nondifferentiable points $a_{j}^{(k)}$. We still denote $C^{2}$ approximation of $\theta_{k}$ by $\theta_{k}$.

$3^{\circ}$. We approximate the nondecreasing $C^{2}$ function $\theta_{k}$ by a $C^{2}$ function whose derivative is always positive. Let $\beta \in C^{2}(\mathbb{R})$ be a bounded $C^{2}$ function with $\beta^{\prime}>0$. If we set

$$
\bar{\theta}_{k}(t)=\theta_{k}(t)+\beta(t) / k,
$$

then this $\bar{\theta}_{k}$ converges to $\theta$ uniformly in $\mathbb{R}$ as $k \rightarrow \infty$. Since $\bar{\theta}_{k}$ is a $C^{2}$ increasing function with $\bar{\theta}_{k}^{\prime}>0$, this completes the proof.

Proof of Theorem 5.2. We may assume that $A$ is bounded and $u$ is bounded in $A$ since our problem is local. Let $u^{\varepsilon}$ be the sup convolution of $u^{*}$ in Lemma 3.5. By Lemmas 3.5 and 3.6 we see $u^{\varepsilon}$ is semiconvex in $A$ and Lipschitz continuous on $\bar{A}$, and is a subsolution of

$$
E_{\varepsilon}\left(y, u^{\varepsilon}, D u^{\varepsilon}, D^{2} u^{\varepsilon}\right)=0 \text { in } A^{\varepsilon} .
$$

Let $\theta_{k}$ be an approximation of $\theta$ in Lemma 5.4. By Lemma 5.3 we observe that $\theta_{k}\left(u^{\varepsilon}\right)$ is also a subsolution of (5.9). Since the convergence $u^{\varepsilon} \downarrow u^{*}$ is monotone and $u^{*}$ is u.s.c., applying Dini's theorem we see $u^{\varepsilon}$ converges to $u^{*}$ uniformly in $\bar{A}$. This implies that $\theta_{k}\left(u^{\varepsilon}\right)$ converges to $\theta\left(u^{*}\right)$ uniformly in $\bar{A}$. We now apply the stability Proposition 2.4 and conclude that $\theta\left(u^{*}\right)$ is a subsolution of (5.2) since $\lim _{*_{\varepsilon} \downarrow 0} E_{\varepsilon} \geq E_{*}$. Since $\theta(u)^{*}=\theta\left(u^{*}\right)$, it follows that $\theta(u)$ is a subsolution of (5.2). q.e.d.

We now give a version of Theorem 5.2 for a parabolic equation

$$
E\left(y, u, D u, D^{2} u\right)=u_{t}+F\left(t, x, u, \nabla u, \nabla^{2} u\right)=0,
$$


where $y=(t, x), \quad D=\left(\partial_{t}, \nabla\right)$, and $F:(0, T] \times W \rightarrow \mathbb{R}$ with $W \subset$ $J\left(\mathbb{R}^{n}\right)$. It turns out that the word "geometric" is consistently used in both the Introduction and Definition 5.2.

Proposition 5.5. Suppose that $E$ is expressed as in (5.10). Then the equation $E=0$ is geometric if (and only if) $F$ satisfies

$$
F=F(t, x, r, p, X) \text { is independent of } r,
$$

and

$$
F(t, x, \lambda p, \lambda X+\sigma p \otimes p)=\lambda F(t, x, p, X)
$$

for $\lambda>0$ and $\sigma \in \mathbb{R}$, whenever each term is well defined.

If $F$ satisfies (5.11a) and (5.11b), we say $F$ is geometric as in the Introduction.

The proof is straightforward from the definitions and is thus omitted.

Theorem 5.6 (Parabolic version). Let $\Omega$ be an open set in $\mathbb{R}^{n}$ and $T>0$. Let $W$ be a dense subset of $J(\Omega)=\Omega \times \mathbb{R} \times \mathbb{R}^{n} \times S^{n \times n}$. Suppose that $F$ is degenerate elliptic and geometric in $(0, T] \times W$. If $u$ is a locally bounded viscosity sub- (super-) solution of $(5.10)$ in $\Omega_{T}=(0, T] \times \Omega$, then so is $\theta(u)$ whenever $\theta: \mathbb{R} \rightarrow \mathbb{R}$ is a continuous nondecreasing function.

Proof. Let $E$ be as in (5.10). By assumptions of $F$ we see $E$ is degenerate elliptic and $E=0$ is geometric. Applying Theorem 5.2 with $A=$ int $\Omega_{T}$ shows that $\theta(u)$ is a sub- (super- resp.) solution of (5.10) in int $\Omega_{T}=(0, T) \times \Omega$. This together with the next lemma implies that $\theta(u)$ is a sub- (super- resp.) solution of (5.10) in $\Omega_{T}$.

Lemma 5.7. If $u$ is a subsolution in $(0, T) \times \Omega$ of (5.10), then $u$ is a subsolution in $\Omega_{T}=(0, T] \times \Omega$ of $(5.10)$.

Proof. The proof is similar to that of Lemma 3.1 (admitting Proposition 3.2). We may assume that $u^{*}-\phi$ attains its maximum over $\Omega_{T}$ at $(T, \bar{x}), \bar{x} \in \Omega$, where $\phi \in C^{2}\left(\Omega_{T}\right)$. As in the proof of Lemma 3.1 we shift $\phi$ by $\phi_{\alpha}$, and find a sequence $\left(t_{\alpha}, x_{\alpha}\right) \in \operatorname{int} \Omega_{T}$ such that $u^{*}-\phi_{\alpha}$ attains its maximum at $\left(t_{\alpha}, x_{\alpha}\right)$ and that $\left(t_{\alpha}, x_{\alpha}\right) \rightarrow(T, \bar{x})$. Since $u$ is a subsolution of (5.10) in int $\boldsymbol{\Omega}_{T}$, passing to the limit in

$$
E_{*}\left(y_{\alpha}, u^{*}\left(y_{\alpha}\right), D \phi\left(y_{\alpha}\right), D^{2} \phi\left(y_{\alpha}\right)\right) \leq 0, \quad y_{\alpha}=\left(t_{\alpha}, x_{\alpha}\right),
$$

yields

$$
E_{*}\left(\bar{y}, u^{*}(\bar{y}), D \phi(\bar{y}), D^{2} \phi(\bar{y})\right) \leq 0, \quad \bar{y}=(T, \bar{x}) .
$$

Remark 5.8. In [24, Proposition 2.2] there is a proof of Lemma 5.7. Our proof is different from that in [24].

We conclude this section by listing examples of geometric, degenerate parabolic equations. We shall suppress the word "degenerate" (because all geometric equations are degenerate). 
Example 5.9. The mean curvature flow equation as well as its generalization

$$
u_{t}-|\nabla u| \operatorname{div}(\nabla u /|\nabla u|)-\nu|\nabla u|=0, \quad \nu \in \mathbb{R},
$$

is a geometric parabolic equation. Indeed, (5.12) is expressed as

$$
u_{t}+F\left(\nabla u, \nabla^{2} u\right)=0
$$

with

$$
F(p, X)=-\operatorname{trace}((I-\bar{p} \otimes \bar{p}) X)-\nu|p|, \quad \bar{p}=p /|p| .
$$

A calculation shows

$$
\begin{aligned}
F(\lambda p, \lambda X+\sigma p \otimes p) & =-\operatorname{trace}((I-\bar{p} \otimes \bar{p})(\lambda X+\sigma p \otimes p))-\lambda \nu|p| \\
& =\lambda F(p, X)-\sigma \operatorname{trace}((I-\bar{p} \otimes \bar{p}) p \otimes p) .
\end{aligned}
$$

Since $(\bar{p} \otimes \bar{p})(p \otimes p)=p \otimes p$, the last term disappears so $F$ satisfies $(5.11 \mathrm{a}, \mathrm{b})$. By Proposition 5.5 we see $(5.12)$ is geometric. Since

$$
-\operatorname{trace}((I-\bar{p} \otimes \bar{p}) Y) \leq 0 \text { for } Y \geq O,
$$

we see $F$ is degenerate elliptic, i.e.,

$$
F(p, X+Y) \leq F(p, X) \text { for } Y \geq O \text {. }
$$

We thus conclude (5.12) is a geometric parabolic equation.

Example 5.10 (An anisotropic version of (5.12)). We consider

$$
u_{t}-|\nabla u| \sum_{i=1}^{n} \frac{\partial}{\partial x_{i}}\left(\frac{\partial H}{\partial p_{i}}\left(\frac{\nabla u}{|\nabla u|}\right)\right)-\beta\left(\frac{\nabla u}{|\nabla u|}\right)|\nabla u|=0
$$

where $H \in C^{2}\left(\mathbb{R}^{n} \backslash\{0\}\right)$ is convex and positively homogeneous of degree 1, i.e.,

$$
H(\lambda p)=\lambda H(p) \text { for } \lambda>0, p \in \mathbb{R}^{n} \backslash\{0\} .
$$

If $H(p)=|p|$ and $\beta=\nu,(5.14)$ is the same as (5.12). (5.14) is a geometric parabolic equation. Indeed, (5.14) is expressed as (5.13) with

$$
F(p, X)=-\operatorname{trace}(A(\bar{p})(I-\bar{P} \otimes \bar{p}) X)-\beta(\bar{p})|p|
$$

and $n \times n$ matrix

$$
A(\bar{p})=\left(\frac{\partial^{2} H}{\partial p_{i} \partial p_{j}}(\bar{p})\right), \quad \bar{p}=\frac{p}{|p|} .
$$

As in Example 5.9 we easily observe that $F$ is geometric. The convexity of $H$ yields $A(\bar{p}) \geq 0$ which implies that $F$ is degenerate elliptic. We 
thus conclude that (5.14) is a geometric parabolic equation. We remark that $(5.15)$ is also expressed as

$$
F(p, X)=-\operatorname{trace}(A(\bar{p}) X)-\beta(\bar{p})|p| .
$$

Indeed, the homogeneity of $H$ implies

$$
H=\sum_{i=1}^{n} \frac{\partial H}{\partial p_{i}} p_{i}
$$

Differentiating this identity in $p_{j}$, we easily see $A(p) p \otimes p=0$.

The anisotropic version of $(5.12)$ is important in studying anisotropic phase transition phenomena such as crystal growth. We refer to [13] for its background.

\section{Existence and uniqueness of solutions}

In this section, as applications of results in $\S 4$ we construct a unique continuous viscosity solution of the initial value problem for a geometric parabolic equation

$$
\begin{gathered}
u_{t}+F\left(t, \nabla u, \nabla^{2} u\right)=0 \quad \text { in } \mathbb{R}_{T}^{n}=(0, T] \times \mathbb{R}^{n}, \\
u(0, x)=a(x)
\end{gathered}
$$

for $a \in C_{\alpha}\left(\mathbb{R}^{n}\right)$, i.e., $a-\alpha$ is continuous with compact support in $\mathbb{R}^{n}$, where $\alpha \in \mathbb{R}$. We also establish a comparison theorem as well as uniqueness of solutions. Since $\mathbb{R}_{T}^{n}$ is unbounded, we shall reduce our problem to the case where the domain is bounded by using "barriers" so that the results in $\S 4$ are applicable. To show the existence of solutions we construct suband supersolutions of the initial value problem (6.1)-(6.2). This leads to the existence of solutions of (6.1)-(6.2) by Perron's method as in Theorem 4.5.

We begin by constructing sub- and supersolutions of (6.1). In what follows we shall always assume that $F=F(t, x, p, X)$ is continuous and degenerate elliptic in $(0, T] \times \mathbb{R}^{n} \times\left(\mathbb{R}^{n} \backslash\{0\}\right) \times S^{n \times n}$.

Lemma 6.1. Suppose that $F$ is geometric and that

$$
\begin{gathered}
F_{*}(t, x, p,-I) \leq c_{-}(|p|), \\
F^{*}(t, x, p, I) \geq-c_{+}(|p|)
\end{gathered}
$$

for some $c_{ \pm}(\sigma) \in C^{1}[0, \infty)$ and $c_{ \pm}(\sigma) \geq c_{0}>0$ with some constant $c_{0}$. We set

$$
u^{ \pm}(t, x)= \pm\left(t+w_{ \pm}(\rho)\right), \quad \rho=|x|, \text { with } w_{ \pm}(\rho)=\int_{0}^{\rho} \frac{\sigma}{c_{ \pm}(\sigma)} d \sigma
$$

Then $u^{-}\left(u^{+}\right.$resp.) is a $C^{2}$ sub-(super-resp.) solution of (6.1) in $\mathbb{R} \times \mathbb{R}^{n}$. 
Proof. We only show that $u^{-}$is a subsolution of (6.1) when (6.3_) holds, since the proof for $u^{+}$is parallel. By definition (6.4), $u=u^{-} \in$ $C^{2}\left(\mathbb{R} \times \mathbb{R}^{n}\right)$. Since $F_{*}$ is geometric and

$$
\begin{gathered}
\nabla w_{-}(\rho)=w_{-}^{\prime}(\rho) \nabla \rho, \\
\nabla^{2} w_{-}(\rho)=w_{-}^{\prime \prime}(\rho) \nabla \rho \otimes \nabla \rho+w_{-}^{\prime}(\rho) \nabla^{2} \rho, \\
\nabla^{2} \rho=\rho^{-1}(I-\nabla \rho \otimes \nabla \rho)
\end{gathered}
$$

with $w_{-}^{\prime}(\rho)>0$, a calculation yields

$$
\begin{aligned}
F_{*}\left(t, x, \nabla u, \nabla^{2} u\right) & =F_{*}\left(t, x,-w_{-}^{\prime}(\rho) \nabla \rho,-w_{-}^{\prime}(\rho) \rho^{-1} I\right) \\
& =w_{-}^{\prime}(\rho) \rho^{-1} F_{*}(t, x,-\rho \nabla \rho,-I),
\end{aligned}
$$

which together with $(6.4)$ gives

$$
u_{t}+F_{*}\left(t, x, \nabla u, \nabla^{2} u\right)=-1+F_{*}(t, x,-x,-I) / c_{-}(\rho),
$$

since $\rho \nabla \rho=x$. Thus the assumption (6.3_) implies

$$
u_{t}+F_{*}\left(t, x, \nabla u, \nabla^{2} u\right) \leq 0,
$$

which means that $u=u_{-}$is a subsolution of (6.1).

Example 6.2. If $F(t, x, p, X)$ is of degree one in $X$ and independent of $t$ and $x$, then $F$ has a form

$$
F(p, X)=-\operatorname{trace}(A(p) X)+B(p) .
$$

If $F$ is geometric and continuous in $p \in \mathbb{R}^{n} \backslash\{0\}$, we see easily that $F$ satisfies $\left(6.3_{ \pm}\right)$by taking

$$
c_{ \pm}(\rho)=\sup _{|p|=1} \operatorname{trace} A(p)+\rho \sup _{|p|=1}|B(p)| .
$$

In particular, (5.12) and (5.14) in Examples 5.9 and 5.10 satisfy $\left(6.3_{ \pm}\right)$. When (6.1) is the mean curvature flow equation (5.12) with $\nu=0$,

$$
F(p, X)=-\operatorname{trace}((I-\bar{p} \otimes \bar{p}) X), \quad \bar{p}=p /|p|,
$$

we have

$$
u^{ \pm}(t, x)= \pm\left(t+|x|^{2} / 2(n-1)\right) .
$$

Moreover, $u^{ \pm}$is a solution of (5.12) with $\nu=0$.

Lemma 6.3. Suppose that $F$ is geometric and that $\left(6.3_{-}\right)\left(\left(6.3_{+}\right)\right.$resp.) holds. For $u^{ \pm}$we set

$$
U_{\xi h}^{ \pm}(t, x)=h\left(u^{ \pm}(t, x-\xi)\right), \quad \xi \in \mathbb{R}^{n},
$$


where $u^{ \pm}$is defined in Lemma 6.1, and $h$ is a continuous nondecreasing function in $\mathbb{R}$. Then $U_{\xi h}^{-}\left(U_{\xi h}^{+}\right.$resp.) is a sub-(super- resp.) solution of (6.1) in $\mathbb{R} \times \mathbb{R}^{n}$.

Proof. By a translation of the variable $x$, Lemma 6.1 implies that $u^{-}(t, x-\xi)$ is a subsolution of (6.1). Since $F$ is geometric, by Theorem 5.6

$$
U_{\xi h}^{-}=h\left(u^{-}(t, x-\xi)\right)
$$

is again a subsolution of (6.1). The proof for $U_{\xi h}^{+}$is parallel so is omitted.

Proposition 6.4. Suppose that $F$ is geometric and that (6.3_) $\left(\left(6.3_{+}\right)\right.$ resp.) holds. Then for every $a \in C\left(\mathbb{R}^{n}\right)$ there is a lower (upper resp.) semicontinuous sub-(super- resp.) solution $v^{-}\left(v^{+}\right.$resp.) of $(6.1)$ in $[0, \infty) \times \mathbb{R}^{n}$ satisfying

$$
v^{-} \leq a \leq v^{+} \text {for } t \geq 0 \text { and } v^{ \pm}=a \text { at } t=0 .
$$

Proof. We construct only a subsolution $v^{-}$of (6.1)-(6.2) by using $U_{\xi h}^{-}$ since a supersolution $v^{+}$of $(6.1)-(6.2)$ is constructed parallelly from $U_{\xi h}^{+}$. Since $u^{-}(t, x)$ is decreasing in $|x|$ and $t$, for each $\xi \in \mathbb{R}^{n}$ the continuity of $a$ guarantees that there is a continuous nondecreasing function $h=$ $h_{\xi}: \mathbb{R} \rightarrow \mathbb{R}$ with $h(0)=a(\xi)$ such that $U_{\xi h}^{-}(t, x) \leq a(x)$ for $t \geq 0$. Since $U_{\xi h}^{-}$is a subsolution of (6.1) in $\mathbb{R} \times \mathbb{R}^{n}$, by Proposition 2.2 the function

$$
v^{-}(t, x)=\sup \left\{U_{\xi h}^{-}(t, x) ; h=h_{\xi}, \xi \in \mathbb{R}^{n}\right\} \leq a(x)
$$

is again a subsolution of $(6.1)$ in $[0, \infty) \times \mathbb{R}^{n}$. Since $h_{\xi}(0)=a(\xi)$ and $U_{\xi h}^{-}(0, x) \leq a(x)$ with $h=h_{\xi}$, we observe that $v^{-}(0, x)=a(x)$ so $v^{-}$ satisfies (6.2). The continuity of $U_{\xi h}^{-}$implies that $v^{-}$is l.s.c.

We next introduce "barriers" to handle the unbounded domain $\mathbb{R}^{n}$.

Lemma 6.5. For $\omega \geq 0$ we set

$$
\psi^{ \pm}(t, x)= \begin{cases}\mp(|x|-\omega t)^{4} & \text { if }|x|>\omega t \\ 0 & \text { otherwise. }\end{cases}
$$

Suppose that $F$ is degenerate elliptic and satisfies

$$
\begin{gathered}
F_{*}(t, x, p, O) \leq \nu_{-}|p|, \\
F^{*}(t, x, p, O) \geq-\nu_{+}|p|
\end{gathered}
$$

with $\nu_{ \pm} \geq 0$ independent of $t, x$, and $p$. Then $\psi^{-}\left(\psi^{+}\right.$resp. $)$is a $C^{2}$ sub-(super-resp.) solution of (6.1) in $\mathbb{R} \times \mathbb{R}^{n}$ provided that $\omega \geq \nu_{-}\left(\omega \geq \nu_{+}\right.$ resp.). 
Proof. Again we only show that $\psi^{-}$is a subsolution of (6.1). Clearly, $\psi^{-}$is $C^{2}$. Since $\psi=\psi^{-}$is convex and $F$ is degenerate elliptic, by (6.6_) we obtain

$$
F_{*}\left(t, x, \nabla \psi, \nabla^{2} \psi\right) \leq F_{*}(t, x, \nabla \psi, O) \leq \nu_{-}|\nabla \psi| .
$$

A calculation shows that $\psi=\psi^{-}$solves $\psi_{t}+\omega|\nabla \psi|=0$. If $\omega \geq \nu_{-}$, this yields

$$
\psi_{t}+F_{*}\left(t, x, \nabla \psi, \nabla^{2} \psi\right) \leq \psi_{t}+\nu_{-}|\nabla \psi| \leq 0
$$

Proposition 6.6. Suppose that $F$ is geometric and degenerate elliptic and satisfies (6.3_) $\left(\left(6.3_{+}\right)\right.$resp.). Then $F$ satisfies $\left(6.6_{-}\right)\left(\left(6.6_{+}\right)\right.$resp.) with $\nu_{ \pm}=c_{ \pm}(1)$.

Proof. If $F$ is degenerate elliptic, (6.3_) implies $F(t, x, p, O) \leq$ $c_{-}(|p|)$. Since $F$ is geometric, we see

$$
F(t, x, p, O)=|p| F(t, x, \bar{p}, O) \leq|p| c_{-}(1), \quad \bar{p}=p /|p|,
$$

which yields $\left(6.6_{-}\right)$with $\nu_{-}=c_{-}(1)$. The estimate $\left(6.6_{+}\right)$is parallelly proved. q.e.d.

We now state the uniqueness and comparison of viscosity solutions of (6.1)-(6.2) in $\mathbb{R}_{T}^{n}$. By $f \in C_{\alpha}(A)$ we mean that $f \in C(A)$ and $f-\alpha$ has a compact support in $A$, where $\alpha \in \mathbb{R}$.

Theorem 6.7 (Uniqueness and comparison). Let $T>0$. Assume that $F(t, p, X)$ is continuous in $(0, T] \times\left(\mathbb{R}^{n} \backslash\{0\}\right) \times S^{n \times n}$ and is geometric and degenerate elliptic, and that $F$ satisfies $\left(6.6_{ \pm}\right)$and

$$
-\infty<F_{*}(t, 0, O)=F^{*}(t, 0, O)<\infty .
$$

Then for a $\in C_{\alpha}\left(\mathbb{R}^{n}\right)$ there is at most one viscosity solution $u_{a} \in$ $C_{\alpha}\left([0, T] \times \mathbb{R}^{n}\right)$ of $(6.1)-(6.2)$. Moreover, if $b \geq a$ with $b \in C_{\beta}\left(\mathbb{R}^{n}\right)$ for some $\beta \geq \alpha$, then $u_{b} \geq u_{a}$ in $\mathbb{R}_{T}^{n}$.

Proof. We may assume $\alpha=0 \leq \beta$. For $\psi^{ \pm}$in (6.5) we set

$$
f_{R}=\min \left(\psi^{-}-R^{4}, 0\right), \quad g_{R}=\max \left(\psi^{+}+R^{4}, \beta\right),
$$

where $\omega \geq \nu_{ \pm}$and $R>0$. We take $R$ large enough so that $f_{R} \leq a(x) \leq$ $b(x) \leq g_{R}$ holds at $t=0$. By Lemma $6.5, \psi^{-}$and $\psi^{+}$are, respectively, sub- and supersolutions of (6.1) in $\mathbb{R}_{T}^{n}$. Since $F$ is geometric and the functions

$$
\theta_{-}(\sigma)=\min \left(\sigma-R^{4}, 0\right), \quad \theta_{+}(\sigma)=\max \left(\sigma+R^{4}, \beta\right)
$$

are continuous nondecreasing functions, by Theorem 5.6 we conclude that $f_{R}=\theta_{-}\left(\psi^{-}\right)$and $g_{R}=\theta_{+}\left(\psi^{+}\right)$are, respectively, sub- and supersolutions 
of (6.1) in $\mathbb{R}_{T}^{n}$. Take $R_{1}$ such that $u_{a}, u_{b}-\beta, f_{R}, g_{R}-\beta$ are supported in $[0, T] \times B\left(R_{1}\right)$, where $B\left(R_{1}\right)$ denotes the open ball of radius $R_{1}$ centered at the origin. Applying the comparison Theorem 4.1 with $\Omega=B\left(R_{1}\right)$ yields $u_{b} \geq u_{a}$. This in particular deduces the uniqueness of $u_{a}$ for a given $a \in C_{\alpha}\left(\mathbb{R}^{n}\right)$.

Theorem 6.8 (Global existence). Let $T>0$. Assume that $F(t, p, X)$ is continuous in $(0, T] \times\left(\mathbb{R}^{n} \backslash\{0\}\right) \times S^{n \times n}$ and is geometric and degenerate elliptic, and that $F$ satisfies (6.3 $)$ and (6.7). Then for $a \in C_{\alpha}\left(\mathbb{R}^{n}\right)$ there is a (unique) viscosity solution $u_{a} \in C_{\alpha}\left([0, T] \times \mathbb{R}^{n}\right)$ of $(6.1)-(6.2)$.

Proof. We may assume $\alpha=0$. Since $\left(6.3_{ \pm}\right)$implies $\left(6.6_{ \pm}\right)$by Proposition 6.6, $f_{R}$ and $g_{R}$ in (6.8) with $\beta=0$ are, respectively, sub- and supersolution of (6.1) in $\mathbb{R}_{T}^{n}$. We take $R$ large so that $f_{R} \leq a(x) \leq g_{R}$ at $t=0$. Let $v^{ \pm}$be sub- and supersolutions of (6.1)-(6.2) constructed in Proposition 6.4, and set

$$
f=\max \left(v^{-}, f_{R}\right), \quad g=\min \left(v^{+}, g_{R}\right) .
$$

Then, by Proposition 2.2, $f$ and $g$ are, respectively, sub- and supersolutions of (6.1)-(6.2) in $\mathbb{R}_{T}^{n}$ and are supported in $[0, T] \times B\left(R_{1}\right)$ for sufficiently large $R_{1}$. Since $f$ and $g$ are, respectively, lower and upper semicontinuous, we now apply the existence Theorem 4.5 with $\Omega=B\left(R_{1}\right)$ to get a solution $u_{a}$ of (6.1)-(6.2) satisfying $f \leq u_{a} \leq g$ supported in $[0, T] \times B\left(R_{1}\right)$. This $u_{a}$ solves $(6.1)-(6.2)$ in $\mathbb{R}_{T}^{n}$ by extending zero outside $B\left(R_{1}\right)$, and satisfies $u_{a} \in C_{\alpha}\left([0, T] \times \mathbb{R}^{n}\right)$.

Remark 6.9. Condition (6.7) follows from $\left(6.6_{ \pm}\right)$if $(t, X) \mapsto$ $F(t, p, X)$ is equicontinuous for small $p$. In particular, if $F(t, p, X)$ is of degree one in $X$ and independent of $t$ as in Example 6.2, all assumptions on $F$ in Theorems 6.7 and 6.8 are fulfilled provided that $F$ is geometric and degenerate elliptic. We thus observe that our Theorems 6.7 and 6.8 are applicable to equations in Examples 5.9 and 5.10. In [5] Theorem 6.7 and a weaker version of Theorem 6.8 are stated.

\section{Evolution of level surfaces}

We now study the $\gamma$-level set $\Gamma(t)$ of the solution $u_{a}$ of (6.1)-(6.2) in Theorems 6.7 and 6.8. Our goal is to show that the $\gamma$-level set

$$
\Gamma(t)=\left\{x \in \mathbb{R}^{n} ; u_{a}(t, x)=\gamma\right\}
$$

and the open set surrounded by $\Gamma(t)$,

$$
D(t)=\left\{x \in \mathbb{R}^{n} ; u_{a}(t, x)>\gamma\right\}, \quad \gamma>\alpha,
$$


are uniquely determined by $(\Gamma(0), D(0))$ and do not depend on a choice of the defining functions $a$ of $(\Gamma(0), D(0))$. In other words, (6.1) can be regarded as an evolution equation of $(\Gamma(t), D(t))$. By the existence Theorem 6.8 we see that there is a unique global evolution family $(\Gamma(t), D(t))(t \geq$ 0 ) for (6.1) with initial data $(\Gamma(0), D(0))$. No regularity of $\Gamma(0)$ is assumed. It turns out that all we need to obtain $(\Gamma(t), D(t))$ is for $D(0)$ to be a bounded open set and for $\Gamma(0)\left(\subset \mathbb{R}^{n} \backslash D(0)\right)$ to be a compact set containing $\partial D(0)$. In particular, when (6.1) is the mean curvature flow equation (1.3) we construct a whole unique evolution family $\Gamma(t)$ moved by its mean curvature. Since $\Gamma(t)$ may be singular, the mean curvature here is understood in some weak sense. By the comparison Theorem 4.1 we shall also show that $\Gamma(t)$ becomes extinct in finite time provided that $n \geq 2$. This extends a result of Huisken [14] where he proved this fact when $\Gamma(0)$ is a uniformly convex $C^{2}$ hypersurface in $\mathbb{R}^{n}(n \geq 3)$ (see [10] for $n=2$ ).

Theorem 7.1 (Uniqueness). Suppose that $F$ and $u_{a}$ are as in Theorem 6.7 with $a \in C_{\alpha}\left(\mathbb{R}^{n}\right)$. Let $\Gamma(t)$ and $D(t)$ be defined by (7.1)-(7.2). If $\gamma>$ $\alpha$, then the evolution family $(\Gamma(t), D(t))$ for $t \geq 0$ is uniquely determined by $(\Gamma(0), D(0))$ and is independent of $a, \alpha$, and $\gamma$. We call $(\Gamma(t), D(t))$ a solution family of $(6.1)$ with initial data $(\Gamma(0), D(0))$.

To prove Theorem 7.1 we prepare an elementary lemma on the comparison of continuous functions.

Lemma 7.2. Suppose that $a, b \in C(\bar{D})$ are positive in $D$ and vanish on $\partial D$, where $D$ is an open set in $\mathbb{R}^{n}$. Then there exists a continuous (strictly) increasing function $\theta: \mathbb{R} \rightarrow \mathbb{R}$ such that $a(x) \leq \theta(b(x))$ in $D$ with $\theta(0)=0$ provided that either

(i) $D$ is bounded, or

(ii) $D$ is an exterior domain, i.e., $\mathbb{R}^{n} \backslash D$ is a nonempty compact set and $a(x), b(x) \in C_{\alpha}(\bar{D})$ for some $\alpha>0$.

Proof. We prove only that case (i) holds. The proof of case (ii) is similar so is omitted. We set

$$
\begin{aligned}
& a_{1}(r)=\sup \{a(x) ; x \in D, \mathrm{~d}(x, \partial D) \leq r\}, \\
& b_{1}(r)=\inf \{b(x) ; x \in D, \mathrm{~d}(x, \partial D) \geq r\}
\end{aligned}
$$

for $r, 0 \leq r \leq R$, where $R=\sup \{\mathrm{d}(x, \partial D) ; x \in D\}$ and $\mathrm{d}(x, \partial D)$ denotes the distance between $x$ and $\partial D$. Since $a_{1}$ and $b_{1}$ are nondecreasing continuous functions with $a_{1}(0)=b_{1}(0)=0$ and $a_{1}(r)>0$, $b_{1}(r)>0$ for $0<r \leq R$, we see that

$$
\bar{a}(r)=a_{1}(r)+r, \quad \bar{b}(r)=b_{1}(r) r / R
$$


are both continuous increasing functions on $[0, R]$ and satisfy

$$
\bar{a}(r)>0, \quad \bar{b}(r)>0 \quad \text { for } 0<r \leq R, \quad \bar{a}(0)=\bar{b}(0)=0 .
$$

By the definition of $\bar{a}$ and $\bar{b}$ we observe that

$$
0 \leq a(x) \leq \bar{a}(r), \quad 0 \leq \bar{b}(r) \leq b(x) \quad \text { with } r=\mathrm{d}(x, \partial D) .
$$

Since $\bar{b}$ is continuous and increasing, so is its inverse function $\bar{b}^{-1}$. We now set $\theta=\bar{a} \circ \bar{b}^{-1}$ and find $\theta$ is continuous and increasing in $[0, \bar{b}(R)]$. We extend $\theta$ outside $[0, \bar{b}(R)]$ so that $\theta$ is a continuous increasing function on $\mathbb{R}$. Evidently, by (7.3) we obtain $\theta(0)=0$. Since $\bar{a}(r)=\theta(\bar{b}(r))$, $0 \leq r \leq R$, it follows from (7.4) that

$$
a(x) \leq \bar{a}(r)=\theta(\bar{b}(r)) \leq \theta(b(x)) \text { for all } x \in \bar{D} .
$$

Proof of Theorem 7.1. Suppose $b \in C_{\beta}\left(\mathbb{R}^{n}\right)$ and $u_{b} \in C_{\beta}\left([0, T] \times \mathbb{R}^{n}\right)$ solves (6.1) with initial data $b$. We set

$$
\begin{aligned}
\Gamma^{\prime}(t) & =\left\{x \in \mathbb{R}^{n} ; u_{b}(x, t)=\gamma^{\prime}\right\}, \\
D^{\prime}(t) & =\left\{x \in \mathbb{R}^{n} ; u_{b}(x, t)>\gamma^{\prime}\right\}, \quad \gamma^{\prime}>\beta .
\end{aligned}
$$

It suffices to prove

$$
\left(\Gamma^{\prime}(t), D^{\prime}(t)\right)=(\Gamma(t), D(t)) \text { for all } t \geq 0
$$

provided that

$$
\left(\Gamma^{\prime}(0), D^{\prime}(0)\right)=(\Gamma(0), D(0)) .
$$

Since $F$ is geometric, applying Theorem 5.6 we may assume $\gamma^{\prime}=\gamma=0$ and $\alpha=\beta<0$ by a translation and dilation of the dependent variable. By (7.6) we apply Lemma 7.2 and conclude that there are continuous increasing functions $\theta_{1}, \theta_{2}: \mathbb{R} \rightarrow \mathbb{R}$ such that

$$
a(x) \leq \theta_{1}(b(x)), \quad b(x) \leq \theta_{2}(a(x)) \text { for } x \in \mathbb{R}^{n}
$$

with $\theta_{1}(0)=\theta_{2}(0)=\gamma=0$. Since $F$ is geometric, by Theorem 5.6 $\theta_{1}\left(u_{b}\right)$ and $\theta_{2}\left(u_{a}\right)$ are solutions of (6.1) with initial data $\theta_{1}(b)$ and $\theta_{2}(a)$ respectively. Our comparison Theorem 6.7 now yields

$$
u_{a} \leq \theta_{1}\left(u_{b}\right), \quad u_{b} \leq \theta_{2}\left(u_{a}\right) \quad \text { in } \mathbb{R}_{T}^{n} .
$$

This implies (7.5) which completes the proof.

Theorem 7.3 (Existence). Suppose that $F$ is as in Theorem 6.8 for all $T>0$, and that $D(0)$ is a bounded open set and $\Gamma(0)\left(\subset \mathbb{R}^{n} \backslash D(0)\right)$ is a compact set containing $\partial D(0)$. Then there exists a unique solution family $(\Gamma(t), D(t))$ for all $t \geq 0$ of (6.1) with initial data $(\Gamma(0), D(0))$. 
Proof. By the assumptions on $(\Gamma(0), D(0))$, there is a defining function $a \in C_{\alpha}\left(\mathbb{R}^{n}\right), \alpha<0$ such that

$$
\Gamma(0)=\left\{x \in \mathbb{R}^{n} ; a(x)=0\right\}, \quad D(0)=\left\{x \in \mathbb{R}^{n} ; a(x)>0\right\} .
$$

Such $a(x)$ is constructed, for example, by

$$
a(x)= \begin{cases}\mathrm{d}(x, \Gamma(0)) & \text { if } x \in D(0), \\ \max (-\mathrm{d}(x, \Gamma(0)), \alpha) & \text { if } x \notin D(0) .\end{cases}
$$

By Theorem 6.8 there is a unique solution $u_{a}$ of (6.1)-(6.2). We now find a solution family $(\Gamma(t), D(t))$ of (6.1) defined by (7.1)-(7.2) with $\gamma=0$. Its uniqueness follows from Theorem 7.1.

Corollary 7.4. Let $F$ and $(\Gamma(0), D(0))$ be as in Theorem 7.3, and $(\Gamma(t), D(t))$ be the solution family of $(6.1)$ with initial data $(\Gamma(0), D(0))$. If $F$ satisfies

$$
F^{*}(t, p,-I) \geq c>0
$$

with some constant $c$ independent of $t>0$ and $p \in \mathbb{R}^{n} \backslash\{0\}$, then $\Gamma(t)$ and $D(t)$ become empty in finite time.

Proof. As in the proof of Lemma 6.1, a calculation shows that

$$
g(t, x)=-\left(t+\rho^{2} / 2 c\right), \quad \rho=|x|,
$$

is a supersolution of (6.1) since (7.7) holds. For $(\Gamma(0), D(0))$ we take a defining function $a \in C_{\alpha}\left(\mathbb{R}^{n}\right)$ with $\alpha<0$ as in the proof of Theorem 7.3. If $M>0$ is sufficiently large, then $a(x) \leq h(g(0, x))$, where $h(\tau)=\max (\tau+M, \alpha)$. Since $h$ is continuous and nondecreasing, applying Theorem 5.6 we observe that $h(g(t, x))$ is a supersolution of (6.1) in $\mathbb{R}_{T}^{n}$ for every $T>0$. Since $u_{a} \in C_{\alpha}\left([0, T] \times \mathbb{R}^{n}\right)$, definition (7.8) implies that both $u_{a}-\alpha$ and $h(g)-\alpha$ are supported in $[0, T] \times B(R)$ for sufficiently large $R$. We now apply the comparison Theorem 4.1 with $\Omega=B(R)$, and find $u_{a} \leq h(g)$ in $\mathbb{R}_{T}^{n}$ for all $T>0$. Definition (7.8) shows that $h(g)-\alpha=0$ for sufficiently large $t$, say $t>T^{\prime}$. This implies that $u_{a}(x, t) \leq \alpha$ for $t>T^{\prime}$. In particular, $\Gamma(t)$ and $D(t)$ become empty for $t>T^{\prime}$.

Corollary 7.5. Let $(\Gamma(0), D(0))$ be as in Theorem 7.3, and $(\Gamma(t), D(t))$ be the solution family of $(5.14)$ with initial data $(\Gamma(0), D(0))$. Assume that $H$ and $\beta$ in (5.14) satisfy

$$
\inf _{|p|=1} \operatorname{trace} A(p)>0 \text { and } \sup _{|p|=1} \beta(p) \leq 0,
$$

where $A=\nabla^{2} H$. Then $\Gamma(t)$ and $D(t)$ become extinct in finite time. In 
particular, if (5.14) is the mean curvature flow equation (1.3), then $\Gamma(t)$ and $D(t)$ become extinct in finite time provided that $n \geq 2$.

Proof. By (5.16), assumption (7.9) implies (7.7). We also observe that (7.9) holds for the mean curvature equation when $n \geq 2$. Thus applying Corollary 7.4 completes the proof.

\section{References}

[1] U. Abresch \& J. Langer, The normalized curve shortening flow and homothetic solutions, J. Differential Geometry 23 (1986) 175-196.

[2] A. D. Alexandroff, Almost everywhere existence of the second differential of a convex function and some properties of convex surfaces connected with it, Leningrad Gos. Univ. Uchen. Zap. Ser. Mat. Nauk 6 (1939) 3-35. (Russian)

[3] S. Angenent, Parabolic equations for curves on surfaces. II: Intersections, blow up and generalized solutions, CMS-Technical Summary Report \#89-24, Univ. of Wisconsin.

[4] K. A. Brakke, The motion of a surface by its mean curvature, Princeton Univ. Press, Princeton, NJ, 1978.

[5] Y.-G. Chen, Y. Giga \& S. Goto, Uniqueness and existence of viscosity solutions of generalized mean curvature flow equations, Proc. Japan Acad. Ser. A 65 (1989) 207-210.

[6] M. G. Crandall, Semidifferentials, quadratic forms and fully nonlinear elliptic equations of second order, Ann. Inst. H. Poincaré Anal. Non Linéare 6 (1989) 419-435.

[7] P. Dupuis \& H. Ishii, On oblique derivative problems for fully nonlinear second order elliptic PDE's on nonsmooth domain, preprint.

[8] C. Epstein \& M. Weinstein, A stable manifold theorem for the curve shortening equation, Comm. Pure Appl. Math. 40 (1987) 119-139.

[9] L. C. Evans \& J. Spruck, Motion of level sets by mean curvature., I, this issue, pp. 635681.

[10] M. Gage \& R. Hamilton, The heat equation shrinking of convex plane curves, J. Differential Geometry 23 (1986) 69-96.

[11] E. De Giorgi, Convergence problems for functionals and operators, Proc. Internat. Meeting on Recent Methods in Nonlinear Analysis (E. De Georgi et al., eds.), Pitagora Editrice, Bologna, 1979, 131-188.

[12] M. Grayson, The heat equation shrinks embedded plane curves to round points, J. Differential Geometry 26 (1987) 285-314.

[13] M. Gurtin, Towards a nonequilibrium thermodynamics of two-phase materials, Arch. Rational Mech. Anal. 100 (1988) 275-312.

[14] G. Huisken, Flow by mean curvature of convex surfaces into spheres, J. Differential Geometry 20 (1984) 237-266.

[15] _ _ Asymptotic behavior for singularities of the mean curvature flow, J. Differential Geometry 31 (1990) 285-299.

[16] H. Ishii, Perron's method for Hamilton-Jacobi equations, Duke Math. J. 55 (1987) 369384.

[17] _ _ On uniqueness and existence of viscosity solutions of fully nonlinear second order elliptic PDE's, Comm. Pure Appl. Math. 42 (1989) 15-45.

[18] H. Ishii \& P. L. Lions, Viscosity solutions of fully nonlinear second-order elliptic partial differential equations, J. Differential Equations 83 (1990) 26-78.

[19] R. Jensen, The maximum principle for viscosity solutions of fully nonlinear second-order partial differential equations, Arch. Rational Mech. Anal. 101 (1988) 1-27. 
[20] R. Jensen, P. L. Lions \& P. E. Souganidis, A uniqueness result for viscosity solutions of second order fully nonlinear partial differential equations, Proc. Amer. Math. Soc. 102 (1988) 975-978.

[21] J. M. Lasry \& P. L. Lions, A remark on regularization in Hilbert spaces, Israel J. Math. 55 (1986) 257-266.

[22] P. L. Lions, Optimal control of diffusion processes and Hamilton-Jacobi-Bellman equations, Part II: Viscosity solutions and uniqueness, Comm. Partial Differential Equations 8 (1983) 1229-1276.

[23] P. de Mottoni \& M. Schatzman, Evolution géometric d'interfaces, C.R. Acad. Sci. Paris Sér. I Math. 309 (1989) 453-458.

[24] D. Nunziante, Uniqueness of viscosity solutions of fully nonlinear second order parabolic equations with noncontinuous time-dependence, Differential Integral Equations 3 (1990) 77-91.

[25] S. Osher \& J. A. Sethian, Fronts propagating with curvature dependent speed: Algorithms based on Hamilton-Jacobi formulations, J. Computational Phys. 79 (1988) 12-49.

[26] J. A. Sethian, Numerical algorithms for propagating interfaces: Hamilton-Jacobi equations and conservation laws, J. Differential Geometry 31 (1990) 131-161.

HOKKAIDO UNIVERSITY, JAPAN 